\title{
Quantification and analysis of coating surface strains in T-bend tests
}

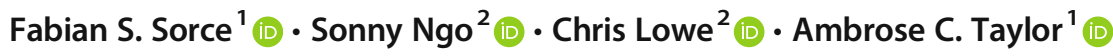

Received: 3 August 2020 / Accepted: 11 January 2021 / Published online: 1 February 2021

(C) The Author(s) 2021

\begin{abstract}
Pre-painted sheet metal (e.g. coil coated with polyester-melamine) undergoes large deformations when formed into architectural cladding or white goods. The coatings provide protection and superior aesthetics, so must withstand failure by cracking or delamination during forming. The T-bend test is an industry standard test used to qualitatively compare the formability of coatings and mimics the conditions experienced during hemming processes. The failure of coatings during forming is strain governed, so understanding the surface strains in the T-bend test is of great interest to manufacturers. For the first time, the maximum surface strains experienced during the T-bend test have been predicted using finite element modelling (FEM) and verified experimentally using digital image correlation. The experimental shapes of the deformed blank are compared with the FEM results for further verification. In addition, a novel analytical model is proposed to determine the maximum surface strains. It is shown that strains of up to $\sim 225 \%$ are applied during a $0 \mathrm{~T}$ test (bent around a zero thickness spacer) reducing to $\sim 23 \%$ at $4 \mathrm{~T}$ (bent around a four times sheet thickness spacer). The finite element model, experimental data and new analytical model show excellent agreement and indicate that behaviour is independent of the substrate thickness or material used. Understanding the strain behaviour quantifies the formerly qualitative T-bend. This will improve the efficacy of the test, allowing metal formers and coating developers to better understand the performance requirements, to reduce waste and to develop better coatings.
\end{abstract}

Keywords Polymer coatings $\cdot$ Forming $\cdot$ Modelling $\cdot$ Digital image correlation (DIC) $\cdot$ Hemming

\section{Introduction}

Assessing the formability of pre-painted metal (PPM) sheet is paramount given the large strains applied during the manufacture of end-products in the construction and domestic appliance industries [1]. The PPM is stored and transported in large coils with masses of up to 25 tonnes before being formed into end-products such as white goods and architectural cladding

Fabian S. Sorce

fabian.sorce12@imperial.ac.uk

Sonny Ngo

sonny.ngo@beckers-group.com

Chris Lowe

chris.lowe@beckers-group.com

Ambrose C. Taylor

a.c.taylor@imperial.ac.uk

1 Department of Mechanical Engineering, Imperial College London, South Kensington Campus, London SW7 2AZ, UK

2 Long Term Development Laboratory, Becker Industrial Coatings Ltd, Goodlass Road, Speke, Liverpool L24 9HJ, UK
[2]. The T-bend test is an industry standard test used to assess the formability and compare the performance of coatings used in PPM. The excellent aesthetics is one of the main benefits of coil coating in addition to the protection of the metal substrate from corrosion and as such, the coatings used must have superior resistance to failure by cracking and delamination.

This work quantifies the surface strains in the T-bend test. The T-bend test is representative of the conditions that a coating would experience during the hemming of PPM and as such, is used to determine the formability of coatings. Coated sheet metal is bent through $180^{\circ}$, varying the radius of curvature of the bend from 0T to 4T (as used by the National Coil Coating Association (NCCA)) by using the sheet itself or spacers, each equivalent to the sheet metal thickness (see Fig. 1) to change the applied strains as defined by the British standard BS EN ISO 17132 [3].

The T-bend test has been historically used as a qualitative test to compare the formability and adhesion of a coating in the form of a go/no-go test. Similarly to the Erichsen cupping test, another industry standard test, it is an empirical test method used in a comparative manner [4]. The T-bend test results are recorded in the form ' $\mathrm{xT}$ ' where the $\mathrm{x}$ denotes the level of T-bend which a coating can withstand without failure by 
Fig. 1 Examples of T-bends a from $0 \mathrm{~T}$ to $2 \mathrm{~T}$ by bending the substrate back on itself and $\mathbf{b}$ from $1 \mathrm{~T}$ to $2 \mathrm{~T}$ using sheet metal spacers (adapted from [3])

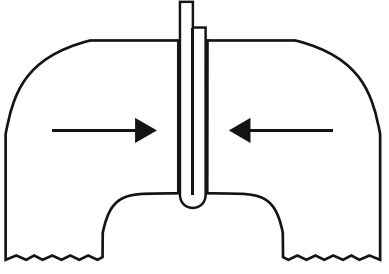

OT

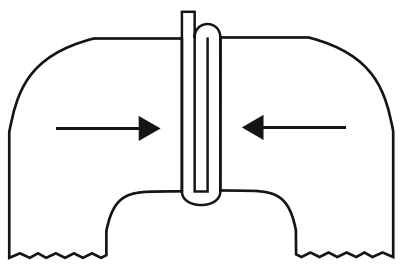

$1 \mathrm{~T}$

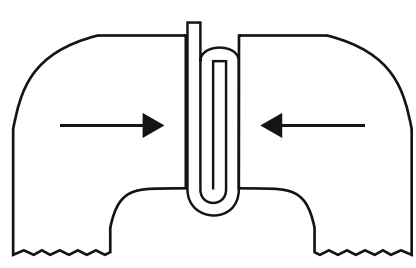

$2 T$

(a)

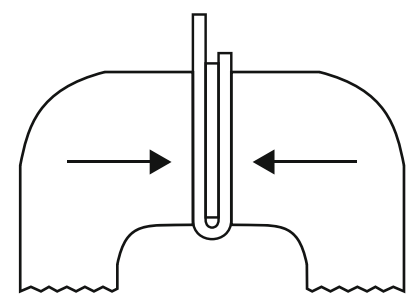

$1 T$

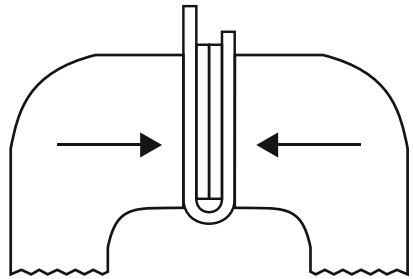

$2 \mathrm{~T}$

(b)

through-thickness cracking of the coating or loss of adhesion. A 0T T-bend refers to a spacer thickness of $0 \mathrm{x}$ the sheet metal thickness, a $1 \mathrm{~T}$ T-bend refers to a spacer thickness of $1 \mathrm{x}$ the thickness of the sheet metal and so on, as used by the NCCA (see Fig. 1). The T-bend level which a coating can withstand without failing by cracking is compared between coatings or a minimum T-bend level which a coating must pass for an application is specified.

The T-bend test has been used to assess the formability of different coating systems in a comparative manner. Yoshida et al. [5] used the T-bend test to assess how crosslinker content affects the formability of polyester-melamine coatings. Sorce et al. [6] investigated the effects of molecular weight in polyester-melamine coatings and showed using the T-bend test that formability increases with an increase in molecular weight. Similarly, Kojima and Watanabe [7] used T-bends and an enamel rater to determine how the molecular weight between crosslinks affects the flexibility of a coating. Giannakopoulos [8] assessed the damage in polyester coatings around their glass transition temperature $\left(\mathrm{T}_{\mathrm{g}}\right)$ during the $\mathrm{T}$ bend test over a 2-h time period, showing that there was viscoelastic strain recovery leading to an increase in damage over time. The damage decreased with an increase in failure strain and essential work of fracture of free films. It is evident that the T-bend test is useful to determine formability trends in coatings but how this translates to practical applications is unclear given the qualitative nature of the test; i.e. it cannot currently be used to assess the maximum failure strain for a particular coating at a given temperature.

The rate of deformation applied in the T-bend test is much higher than the test rate in the Erichsen cupping test $[6,9]$, in addition to the applied surface strains also being much higher
$[10,11]$. It was shown by Ueda et al. [12] that coatings were required to have a free film strain to failure greater than $\sim 200 \%$ to pass a $0 \mathrm{~T} \mathrm{~T}$-bend test despite determining that the applied strain in a $0 \mathrm{~T}$ T-bend test was $\sim 100 \%$. Greunz et al. [11] used T-bend tests to study the adhesion properties of polyestermelamine coatings and found that the level of T-bend which a coating is able to pass is mainly affected by the surface treatment of the hot-dipped galvanised (HDG) steel substrate. The formability of an electroplated chromium coating on a steel substrate was assessed by Zhang et al. [13]. Cross-sections of the T-bends were examined and it was proposed that the larger the compressive strain (determined using the surface strain approximation proposed by Ueda et al. [12]) without interface cracking implies better interfacial adhesion, which shows the wide-ranging applicability and interest in the T-bend test. Kim and Nairn [14,15] studied the cracking process of coatings on substrates during tensile loading and bending. A fracture analysis was proposed regarding the cracking of coatings on a substrate, and this was related experimentally to a fracture toughness. It was found that the substrate had a significant impact on the toughness behaviour of the coatings. Hence, it is clear that coating behaviour is influenced by the substrate's response to deformation. The research presented in the literature demonstrates that the T-bend test is simply used as a means to an end and that there is a knowledge gap in understanding the test which needs to be addressed.

For example, Deflorian et al. [16] and others [12, 17-19] have investigated the effects of mechanical deformation (such as deep drawing in the Erichsen cupping test) on coating performance, demonstrating the interest in being able to understand the performance limitations of a coating on sheet metal. Behrens et al. [18] investigated the reduction of gloss in white 
goods induced by forming processes and proposed a formingrelated gloss-reduction diagram which can be used to limit the amount of gloss reduction by understanding the relationship between gloss reduction and the applied strains. A similar study was performed by Tekkaya et al. [17]. These works suggest that the key to produce more informative testing to improve coating performance requires the test methods used and modes of deformation (e.g. the applied strains) to be understood. The strains in the T-bend test are of particular interest as it has been noted in the literature that failure during the forming of coatings on metal substrates is a strain governed mechanism, as the strain which a given coating is subjected to is equal to that of the substrate surface $[9,10,12,20]$. The failure stress of the PPM is governed by the substrate [10]; hence, improved formability of coatings can be achieved by better understanding the deformation process and what critical forming strain level causes failure of the coating.

This work investigates the surface strains in T-bend tests of HDG steel with a thickness of $0.6 \mathrm{~mm}$ from 0T (no spacer, $0 \mathrm{~mm}$ ) to $4 \mathrm{~T}$ (spacer of 3 sheet thicknesses, $\sim 1.8 \mathrm{~mm}$ ) using the sheet metal spacer method based on the standard [3] (see Fig. 1b). Finite element analysis has been used to predict the surface strains in T-bend tests from $0 \mathrm{~T}$ to $4 \mathrm{~T}$. The finite element (FE) surface strains are validated experimentally using a novel digital image correlation (DIC) technique. A comparison between the predicted and experimentally determined profiles is made to further validate the strain results. A novel, simple analytical model is developed and proposed to accurately capture the strain behaviour in the T-bend tests. These findings quantify the T-bend test and provide an effective method to evaluate coating behaviour in T-bend tests.

\section{Experimental}

\subsection{Materials}

The substrate used was a construction grade hot-dipped galvanised (HDG) sheet steel, primed with a layer of strontium chromate cross-linked polyester-melamine $(\sim 5-7 \mu \mathrm{m}$ thick). Panels measuring $220 \times 150 \mathrm{~mm}^{2}$ with a thickness of $0.6 \mathrm{~mm}$ were used. These were coated with a white $\mathrm{TiO}_{2}$-pigmented polyester-melamine coating, chosen for its good formability, with a thickness of $\sim 20 \mu \mathrm{m}$. The glass transition temperature $\left(\mathrm{T}_{\mathrm{g}}\right.$ ) of the coating was determined to be $28^{\circ} \mathrm{C}$ using differential scanning calorimetry (DSC). Blanks of $25 \times 10 \mathrm{~mm}^{2}$ were cut from the coated panels using a foot-operated guillotine.

\subsection{Finite element modelling}

PAM-STAMP Professional software was used to create a finite element (FE) model to determine the surface strains in the $\mathrm{T}$-bend test from $0 \mathrm{~T}$ to $4 \mathrm{~T}$ based on the $\mathrm{T}$-bend testing standard BS EN ISO 17132:2007 [3] using a 0.6-mm-thick HDG steel blank $(10 \mathrm{~mm}$ wide $\times 30 \mathrm{~mm}$ long (the rolling direction was orientated along the length of the blank)). The material properties (Young's modulus, yield stress, post-yield behaviour, Poisson's ratio, etc.) of the HDG steel were taken from the material library of the modelling software and have been shown to be representative of the substrate used in the experiments $[10,20]$. The coating is not modelled as the strain in a coating is equal to the substrate surface strains $[9,20]$.

The auto-mesh feature in PAM-STAMP was utilised for computational efficiency as the area of interest in the T-bend is solely that of the curved face. This resulted in a final mesh of 2D Belytschko-Tsay 4-node shell elements (with five integrations points through the shell thickness) with characteristic lengths of $\sim 0.078 \mathrm{~mm} \times 0.117 \mathrm{~mm}$ (aspect ratio of 1.5) in the region of interest.

The FE model is comprised of three stages, as shown schematically in Fig. 2. In stage 1, the blank and spacer are positioned and held in place by a clamping force of $1 \mathrm{kN}$ applied to the spacer (see Fig. 2). The clamping force is maintained throughout the simulation. For a 0T T-bend, an infinitesimally small thickness $(0.0001 \mathrm{~mm})$ spacer is used for computational purposes to prevent singularities in the solver from occurring (this would be $0.000167 \mathrm{~T}$ ). The end of the spacer that the substrate is bent around has a radius of curvature equal to half the thickness of the spacer (semi-circular in shape) to ensure a representative bend profile (spacers without curvature were found to distort the bend profile). In stage 2, the blank is bent to $90^{\circ}$ by a press moving in the vertical direction (see Fig. 2). In stage 3 , the blank is bent to $180^{\circ}$ by a curved press moving in the horizontal direction (see Fig. 2).
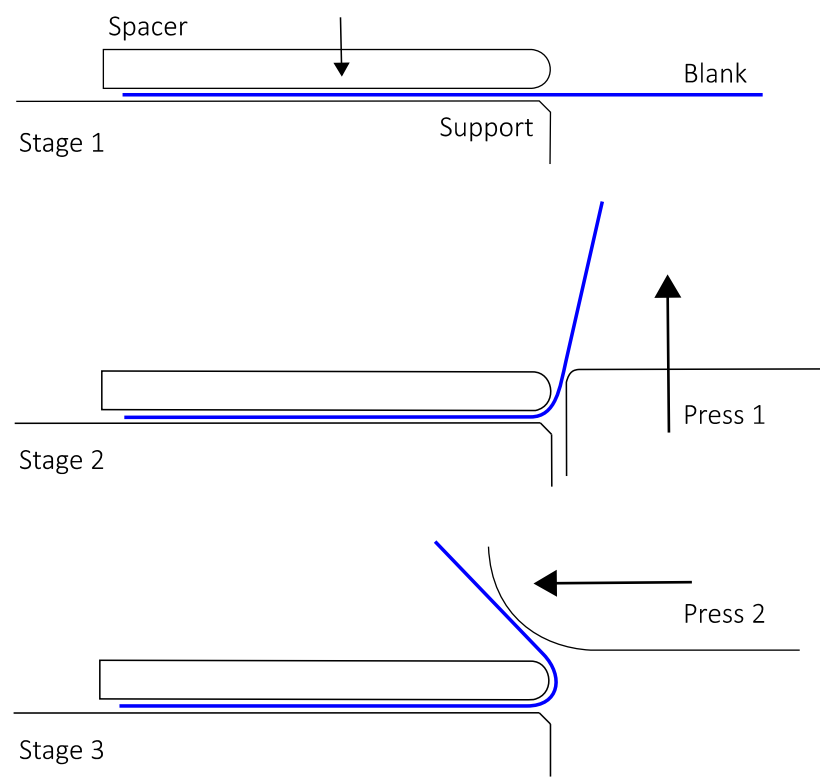

Fig. 2 Schematic showing the three stages of the T-bend test process in the $\mathrm{FE}$ model; stage 1 is clamping, stage 2 is a $90^{\circ}$ bend and stage 3 is a $180^{\circ}$ bend 
In the T-bend test, the bend surface is not observed during the bending process, but only after the final bend to $180^{\circ}$ has been completed. Therefore, only the results of the final bent blank are presented and discussed. The data presented are taken from the outer surface of the mid-plane cross-section (see Fig. 3). The profiles of the T-bends in their final form are predicted and compared to the experimental profiles.

The strains reported are engineering strains, as opposed to true strains. Specifically, the value reported is the engineering major strain (equivalent to the maximum principal strain), which is the largest strain seen in the system and is a result of transforming the coordinate system to take into account the directional nature of the strain. The engineering directional strain (a 1D strain measurement without transformation of the coordinate system to reflect the surface normal of any given point) across the curvature of the T-bend has also been determined as this is assumed to be the most comparable to the measurement which is performed experimentally. The engineering major strains referenced represent the strains on the face of the outer surface on which the coating is present.

The model calculates the strains in a Cartesian coordinate system (see Fig. 8), but strain data in this form cannot be plotted on a conventional $\mathrm{x}-\mathrm{y}$ graph. Therefore, the Cartesian coordinates of the profile of the T-bend are transformed into a linear system where the strains are displayed against their curvilinear coordinates. A schematic of this transformation from Cartesian coordinates to curvilinear coordinates is shown in Fig. 4.

\subsection{Analytical modelling}

Ueda et al. [12] proposed that the maximum strain in the Tbend test could be determined using the assumption that the position and length of the neutral axis of the substrate remain constant throughout the bending process. The bent blank is assumed to have a semi-circular profile and the neutral axis of

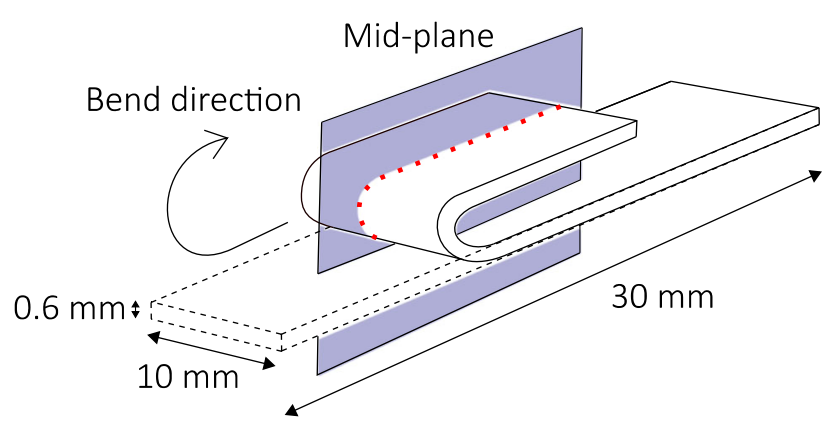

N.B. Not drawn to scale

Fig. 3 Schematic showing the data points analysed at mid-plane in the Tbend test the blank remains at the centre of the thickness, from which an estimate of the outer surface strain $(\varepsilon)$ was proposed as:

$\varepsilon_{\text {Ueda }}=\frac{1}{N+1}$

where $N$ is the number of spacers (i.e. $N=0$ for 0 T). The strains predicted using the Ueda et al. model decrease exponentially as $N$ increases.

Eq. (1) is a simplification of Eq. (2).

$\varepsilon=\frac{2 \pi r_{\mathrm{o}}-2 \pi r_{\mathrm{n}}}{2 \pi r_{\mathrm{n}}}$

where $r_{\mathrm{o}}$ is the outer radius of curvature and $r_{\mathrm{n}}$ is the neutral axis radius of curvature, where:

$r_{\mathrm{o}}=\frac{(N+2)}{2} \cdot t$

$r_{\mathrm{n}}=\frac{(N+1)}{2} \cdot t$

where $t$ is the substrate thickness. Based on the premise that the length of the neutral axis does not change, the strain can be determined by comparing the length of the outer surface with that of the neutral axis (see Eq. (2)). Equations (3) and (4) show how the radii of curvature for the outer surface and neutral axis are functions of $t$ and $N$. The principle used in Eqs. (3) and (4) can also be used to determine the radius of curvature of the spacer and the inner radius $\left(r_{\mathrm{i}}\right)$ of the bend:

$r_{\mathrm{i}}=\frac{N \cdot t}{2}$

An alternative solution was proposed by Wang et al. [21], who investigated the strains experienced by sheet metal during the hemming process (equivalent to a 1T T-bend). The model proposed by Wang et al. assumes an elliptical profile and that the neutral axis remains in the mid-plane of the thickness of the substrate. The strain is calculated using:

$\varepsilon_{\text {Wang }}=\frac{2 \sqrt{3}}{3} \ln \left(1+\frac{t_{0}}{2 \rho_{\mathrm{n}}}\right)$

where $t_{0}$ is the initial thickness of the substrate and $\rho_{\mathrm{n}}$ is the radius of curvature of the neutral axis at the mid-point (apex of the T-bend). The radius of curvature for an ellipse is given by:

$\rho_{\mathrm{n}}=\frac{a^{2}}{b}$

where $a$ is the semi-axis length in the $\mathrm{x}$-direction and $b$ is the semi-axis length in the $y$-direction. In a similar fashion to the Ueda et al. model, this model predicts an exponential decrease in the strains with an increase in the T-bend level. Wang et al. [21] used a numerical solution to determine the value of $b$; 
Fig. 4 a Transformation of the Cartesian coordinate system used in the FE model of the T-bend test to a curvilinear coordinate system. b An example of the midplane of a $0 \mathrm{~T} \mathrm{~T}$-bend profile in Cartesian coordinates and $\mathbf{c}$ an example of the variation in major strain over the T-bend surface at $0 \mathrm{~T}$ versus the curvilinear length

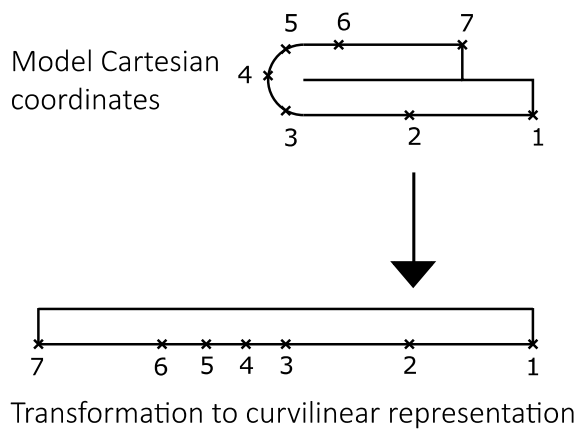

(a)

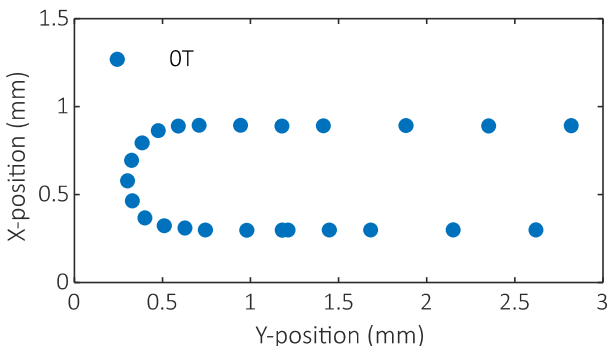

(b)

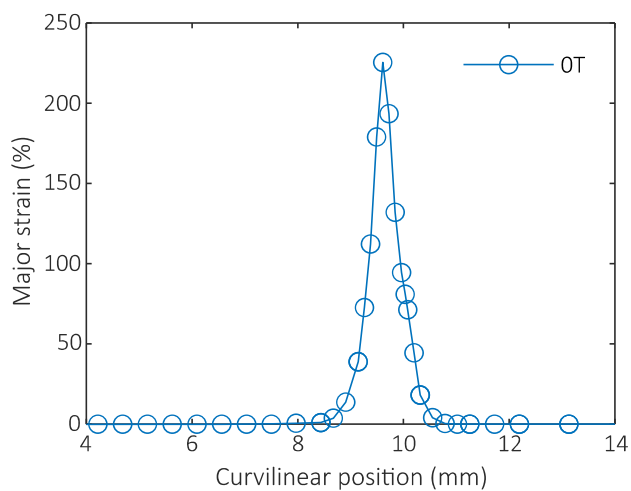

(c) however, as this numerical solution is unavailable, $a$ and $b$ have been determined from the FE predicted profiles.

\subsection{Experimental determination of surface strains}

The surface strains of the T-bend test were determined experimentally using a digital image correlation (DIC) method. Measurement of the surface strains proved difficult due to the area within which the maximum strains occur was so small and because the DIC cameras were unable to observe the area of interest during the process of bending, as the views prior to and after bending are orthogonal views. Therefore, the conventional digital image correlation methods typically used to measure surface strains, e.g. [10], were not appropriate, and hence, a new technique was devised to measure the strains. To reduce cracking in the coating due to the very high strains in a 0T T-bend, all of the T-bends were performed at an elevated temperature of $\sim 34{ }^{\circ} \mathrm{C}$ (i.e. $6{ }^{\circ} \mathrm{C}$ above $\mathrm{T}_{\mathrm{g}}$ ) to increase the failure strain of the coating.

\subsubsection{T-bend test}

The T-bend tests were performed according to BS EN ISO 1732:2007 [3]. Uncoated panels were used as spacers, e.g. using a spacer of $1.8 \mathrm{~mm}$ for a 3T T-bend. An electric oven was set to a temperature of $\sim 34^{\circ} \mathrm{C}$. A pillar drill vice and two polished steel blocks were placed in the oven and left for at least $45 \mathrm{~min}$ to equilibrate. The specimen blank (and spacers if used) was placed in the oven for a minimum of 5 min before commencing the test.

The vice was used to clamp the blank between the two polished steel blocks leaving $10 \mathrm{~mm}$ exposed and the blank was then bent to $90^{\circ}$ with the coating on the outer surface of the bend (see Fig. 5). The specimen was removed and manually further bent to $\sim 135^{\circ}$. The v-shaped blank was reinserted into the vice, which was tightened to complete the T-bend by achieving a bend of $180^{\circ}$.

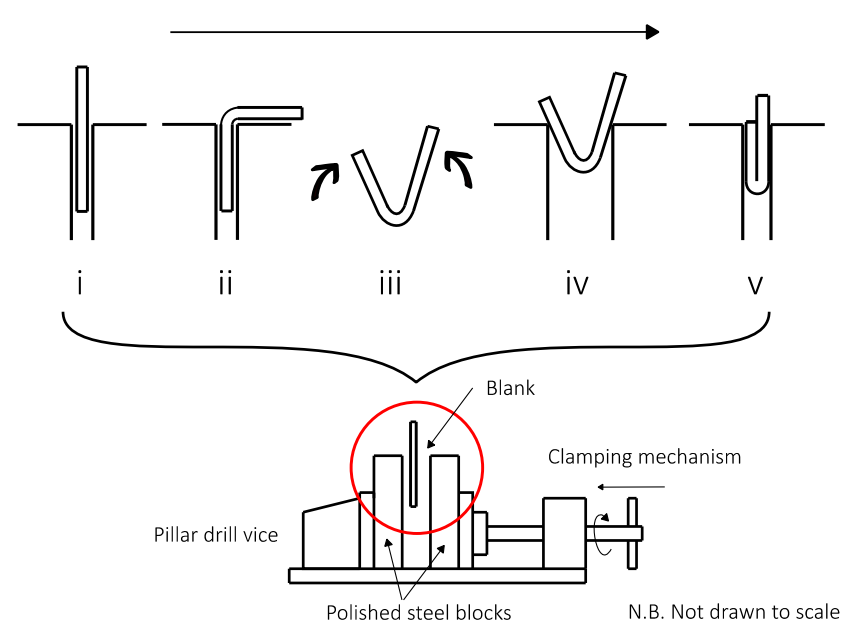

Fig. 5 Schematic depicting the experimental T-bend process 
Similarly, for 1T and higher order T-bends, the same procedure was used with the addition of uncoated panel spacers (e.g. when clamping initially, it would be both the specimen blank and spacer clamped in the vice). This technique was chosen for its flexibility, the ease with which the tests could be performed at a desired temperature and for its comparability to T-bend setups in industry. At least two repeats were performed for each level of T-bend.

\subsubsection{Strain measurement}

A 3.05-mm diameter copper transmission electron microscopy (TEM) support grid with a square 300 mesh (ACC2300C (now AGG2300C) from Agar Scientific) was used to create a grid of squares on the coated substrate. The square mesh was used as a mask and gold sputter coating (thickness $\sim 10 \mathrm{~nm}$, produced by an Agar Scientific Automatic Sputter Coater) was used to create a negative of the grid (see Fig. 6a). A Zeiss Axio Scope A1 Microscope $(5 \times$ lens) fitted with a Leica MC190 HD microscope camera was used to capture the grid pattern in the undeformed state (see Fig. 6a) and after deformation (see Fig. 6b). The Tbend procedure was performed ensuring that the sputter-coated grid was located on the apex of the bend. An image of the apex of the deformed blank was then taken using the microscope. The image brightness and contrast were manually adjusted with Microsoft Photos. Using identifiers in the grid (such as the A symbol) as reference points to index the measurement points, the engineering strain across the face of the bend was determined as a function of pixels using Eq. (8). The strain $(\varepsilon)$ measured is directional across the face of the bend (in the direction of the Xposition axis (see Fig. 4b) parallel to the apex of the bend) and approximates a 2D distance between each measurement point.

$\varepsilon=\frac{\left(x_{\mathrm{i}+1}^{\mathrm{f}}-x_{\mathrm{i}}^{\mathrm{f}}\right)-\left(x_{\mathrm{i}+1}^{0}-x_{\mathrm{i}}^{0}\right)}{x_{\mathrm{i}+1}^{0}-x_{\mathrm{i}}^{0}}$

where $x_{\mathrm{i}}$ and $x_{\mathrm{i}+1}$ are the pair of measurement points. The superscript 0 refers to the original point (before deformation), and the superscript $\mathrm{f}$ refers to the final point (after deformation).

The profiles of each T-bend were captured using a digital single-lens reflex (DSLR) camera. A black permanent marker was used to colour the side profile to increase the contrast when imaging against a white background by highlighting the substrate thickness. The profiles were digitised with Inkscape software to be compared to the profiles predicted by the FE modelling.

The process of image manipulation to determine the strain across the T-bend surface is demonstrated in Fig. 6. Figure 6a shows a representative micrograph of the undeformed grid on the blank substrate before deformation, whilst Fig. 6b shows the deformed grid after a 3T T-bend. The deformation of the grid is clear as the squares are stretched to become rectangles. The curvature of the T-bend can be seen by the blurring of the edges of the T-bend, as the (now parallel) surfaces move beyond the depth of field of the camera. The method of measuring the surface strains presented here is only valid for the strains determined on the peak of the T-bend curve. Due to the curvature of the Tbend surface, when the face of the T-bend is observed, the image viewed can give the impression that the distance between grid points has reduced. This is an optical illusion due to the $3 \mathrm{D}$ geometry being imaged in $2 \mathrm{D}$ and is the result of not viewing the grid perpendicular to the surface of the T-bend except at the apex (see Fig. 7). It is assumed that there is no difference in the vertical direction (out of plane) between adjacent strain measurement points. The typical variation between measurement points in the vertical direction at the apex of the bend is $\sim 0.002 \mathrm{~mm}-$ $0.003 \mathrm{~mm}$ and $\sim 0.03 \mathrm{~mm}-0.04 \mathrm{~mm}$ at the curved edges over a $0.1-\mathrm{mm}$ distance in the $\mathrm{x}$-direction. To negate any effects of variation in the vertical direction at the curved edges, the strains were determined over lengths comparable to the full width at half maximum (FWHM) height of the predicted directional strain distributions (see \$3.3). It should be noted that additional errors due to the focus of the lens are assumed to be negligible as the points used to calculate the highest strain values are in good focus.

Due to low contrast in the deformed images and blurring in the micrographs, computer-generated gridlines were inconsistent in determining the edges of the grid negative. Therefore, digital gridlines, $0.5 \mathrm{px}$ in width, were drawn onto the micrograph using Inkscape over the area of interest, i.e. the central area of the T-bend including the reference point for the strain measurements (the A symbol was typically used as a reference). The gridlines were placed along the edges of the squares. The micrograph layer was then removed leaving a digital grid equal to the grid in the micrograph. Representative digital grids for the undeformed and deformed surfaces are shown in Fig. $6 \mathrm{c}$ and d respectively.

The images of the digital grids were imported into MATLAB whereby a custom-developed code searched for the intersection points of the gridlines and recorded these coordinates as measurement points. The number of measurement points typically formed a matrix of $\sim 14$ by 26 giving a total of $\sim 351$ measurement points resulting in $\sim 173$ strain measurements per test. These intersection points were then sorted into rows of data and plotted as indicated in Fig. 6e and $\mathrm{f}$ for the undeformed and deformed grids respectively. The strains across individual black squares were calculated from the intersection point positions using Eq. (8).

\section{Results and discussion}

\subsection{Introduction}

The shape of the T-bend profiles and the surface strains for Tbends from 0T to 4T have been predicted by FE simulations. 


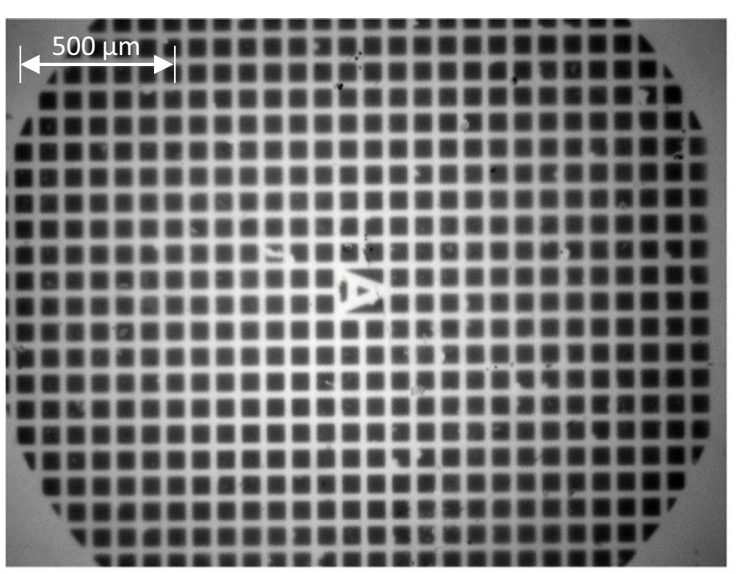

(a)

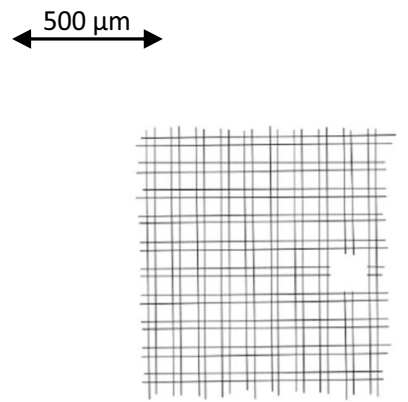

(c)

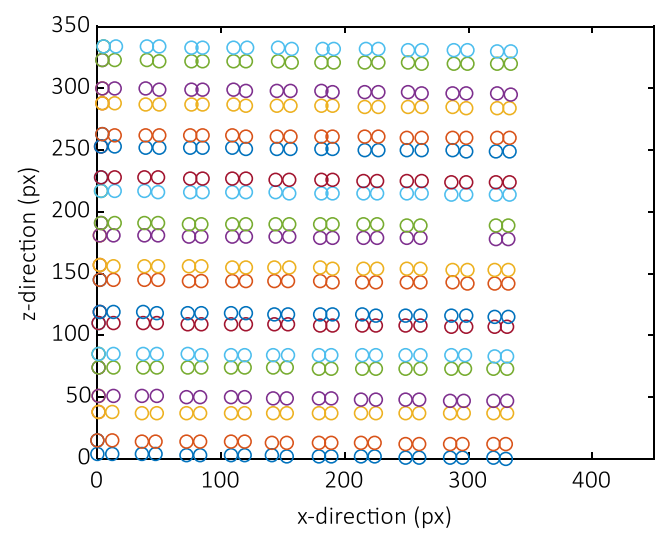

(e)

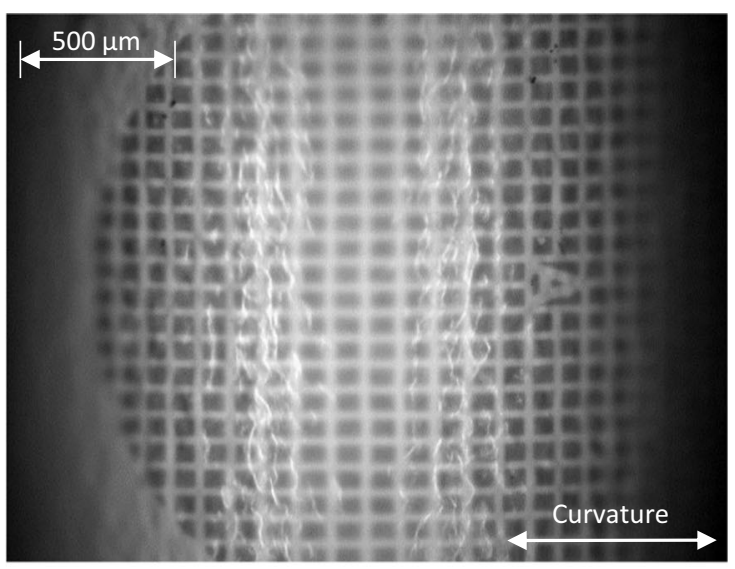

(b)

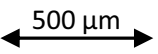

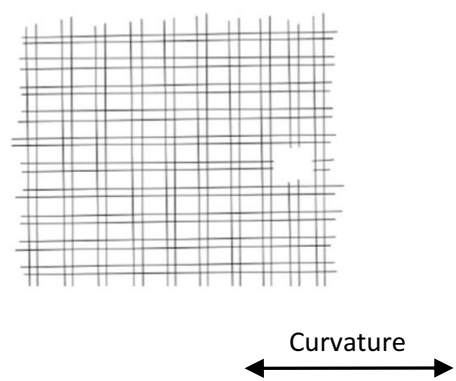

(d)

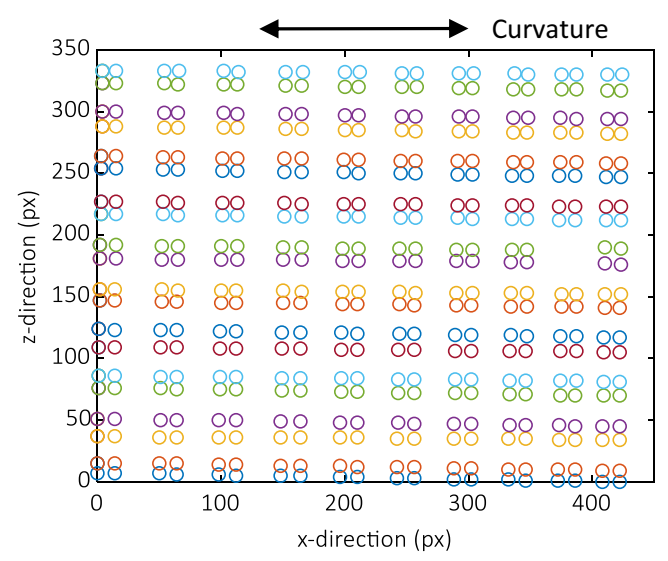

(f)

Fig. 6 Process by which the surface strains of the T-bend are determined, a undeformed negative of the grid, $\mathbf{b}$ deformed negative of the grid, $\mathbf{c}$ undeformed digital gridlines, $\mathbf{d}$ deformed digital gridlines, $\mathbf{e}$ undeformed measurement points and $\mathbf{f}$ deformed measurement points

They have also been predicted analytically based on the work by Ueda et al. [12] and Wang et al. [21]. In addition, a new simple analytical model has been proposed to determine the surface strains. The strains have also been measured experimentally using custom DIC from $0 \mathrm{~T}$ to $3 \mathrm{~T}$ to validate the modelling work.

\subsection{T-bend profiles}

The severity of the surface strains experienced during the Tbend test is dependent on the imposed curvature of the Tbend. As the thickness of the spacer is increased, the radius of curvature increases (curvature decreases) and hence, the 


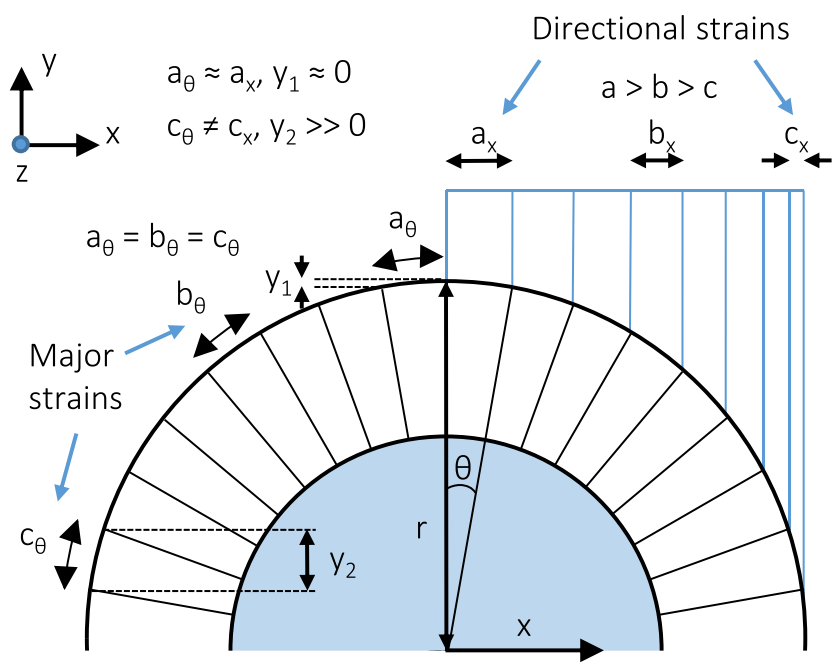

Fig. 7 Schematic demonstrating how viewing the distance between two points on a surface artificially reduces the measured distance if not viewed perpendicularly to the surface, and the directions in which the major and directional strains act

surface strain decreases. The predicted T-bend profiles from the FE model are shown in Fig. 8. The profiles presented are taken at the mid-plane (see Fig. 3) to eliminate any edge effects, and the profile line represents the mid-thickness axis of the substrate. The curve of the T-bend shows a semi-circular profile. The ends of the blank are parallel to each other as they have not been subjected to bending. The top edge of the profile shows a slight asymmetry compared to the lower edge, attributed to clamping effects. As the level of T-bend is increased, there is a clear increase in the radius of curvature. The increase in the radius of curvature is proportional to the increase in the T-bend level given the constant increment in spacer thickness between each T-bend level.

Assuming that the T-bend curvature follows a semicircular path $(a=b)$, an analytical profile has been predicted for each level of T-bend using:

$x=a \cdot \cos \theta$

$y=b \cdot \sin \theta$

where $a$ and $b$ are the semi-axes and $\theta$ is the angle of the profile $(0 \leq \theta \leq \pi)$.

Fig. 8 Comparison of analytically determined (semicircular) and FE-predicted midplane profiles for the T-bend test
The analytical profiles are presented in Fig. 8 and show very good agreement with the FE model profiles. This confirms that it is appropriate to assume a semi-circular profile.

The experimentally determined T-bend profiles are compared to the FE model mid-plane profiles in Fig. 9. Whilst the FE- and analytically predicted T-bend profile results present the mid-thickness axis position, the experimental profile results are derived from the T-bend silhouette. The comparison does not validate the FE model predictions as the full FEpredicted profile is not shown, but the results allow the reader to compare the FE predictions with the measured shape. Note that Fig. 8 showed good agreement between the analytical and FE model predictions. Similarly to the predicted results, the experimental profiles show an increase in the radius of curvature of the T-bends with an increase in the level of T-bend.

The experimental shapes of the outer radius curvature show a semi-circular profile, which is in very good agreement with the FE predictions. However, there is a slight skew of the inner radius profiles towards a semi-elliptical shape which is attributed to edge effects in the substrate. The material at the inner surface is subjected to compressive strains and must deform to accommodate these strains. The compressive strains affect the profile shape at the substrate edges where there are no material constraints causing a slight curling of the material (although it should be noted that these effects are relatively small).

The inner radius of a $0 \mathrm{~T} \mathrm{~T}$-bend is prone to forming a teardrop (see Fig. 9). The teardrop is a result of constraints on the way in which the substrate can deform due to the large applied strains. If the force used is not large enough to induce sufficiently large compressive strains or the material is not ductile enough to compensate and compress, a teardrop will form due to the material flowing and deforming outwards. A $0 \mathrm{~T}$ T-bend teardrop typically has an experimentally determined radius of curvature of $\sim 0.051 \mathrm{~mm}$.

Semi-circular profile approximations agree with the work by Ueda et al. [12], whilst semi-elliptical profiles agree with the work by Wang et al. [21]. The differences in the shapes of the profiles are small, and the observed experimental and FE profile results show good agreement with semi-circular profiles which gives confidence in the strain measurements presented in §3.6. For simplicity, purely semi-circular profiles are assumed in the following sections.

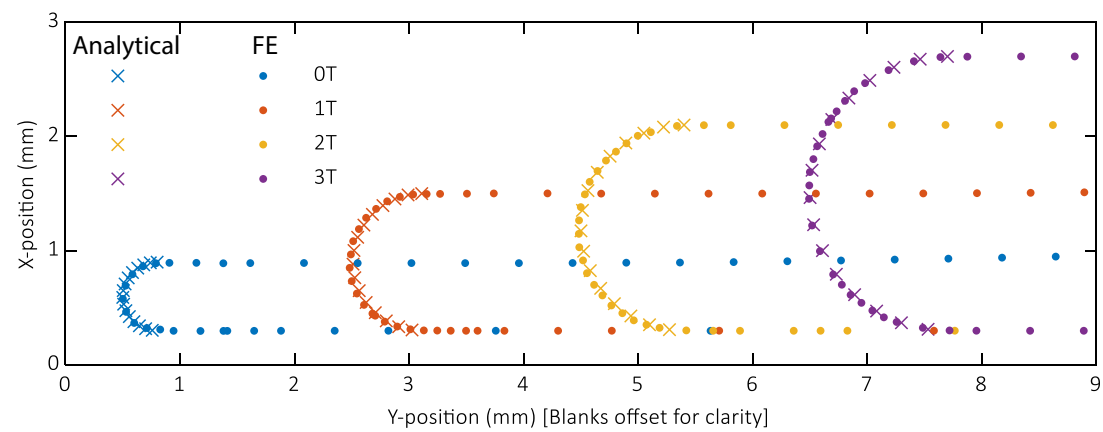


Fig. 9 Comparison of experimentally determined edge profiles and FE-predicted midplane profiles for the T-bend test

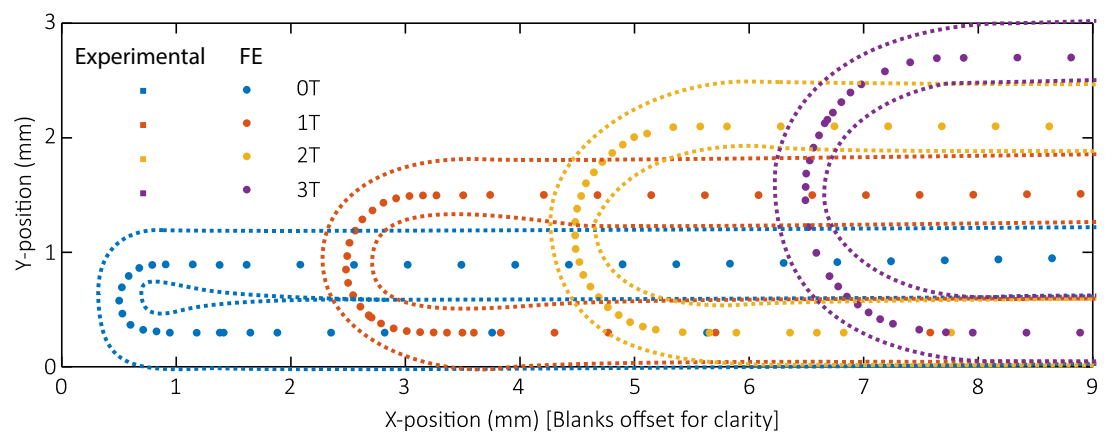

\subsection{FE surface strains in T-bends}

The maximum major and directional surface strains in T-bend tests from 0T to 4T for 0.6-mm-thick HDG steel substrates determined using the FE model are shown in Table 1. In addition to the T-bend levels in increments of one sheet thickness (1T, 2T, 3T, 4T), the strains for fractional thickness $\mathrm{T}$ bends are also included $(0.25 \mathrm{~T}, 0.5 \mathrm{~T}, 0.75 \mathrm{~T}$ and $1.5 \mathrm{~T})$, as the strain decreases rapidly with an increase in spacer thickness at low T-bend levels. The predictions of both types of strain decrease with an increase in the T-bend level. The major strains are higher than the directional strains at low T-bend levels (due to averaging effects in the directional strains) and start to converge at higher T-bend levels. A comparison between the predicted maximum major and directional surface strains versus the T-bend level is shown in Fig. 10. Note that the major strain accounts for the change in direction of the plane of the substrate, i.e. the major strain acts tangentially to the normal of the substrate at any given point whilst the directional strain does not account for this directionality. The behaviour is similar for both types of strain, exhibiting an exponential decrease in strain with an increase in the T-bend level. As noted above, the magnitude of the major strains is larger than the directional strains at low T-bend levels (see Fig. 7). Unless specified otherwise, henceforth FE strain will refer to the major strain.

The maximum absolute minor strains (i.e. the largest compressive strains at the inner radius of the bend) are also shown in Table 1. Similar to the major strain values, as the level of Tbend is increased, the magnitudes of the minor strains

Table 1 Maximum major, directional and minor surface strains predicted by FE for T-bends from 0T to 4T

\begin{tabular}{lllllllllll}
\hline Strain type & \multicolumn{1}{l}{ Maximum strain (\%) } \\
\cline { 2 - 10 } & $0 \mathrm{~T}$ & $0.25 \mathrm{~T}$ & $0.5 \mathrm{~T}$ & $0.75 \mathrm{~T}$ & $1 \mathrm{~T}$ & $1.5 \mathrm{~T}$ & $2 \mathrm{~T}$ & $3 \mathrm{~T}$ & $4 \mathrm{~T}$ \\
\hline Major & 225 & 170 & 120 & 95 & 77 & 58 & 49 & 35 & 27 \\
Directional & 184 & 149 & 114 & 91 & 74 & 56 & 45 & 31 & 26 \\
|Minor| & -69 & -63 & -54 & -48 & -43 & -36 & -32 & -26 & -21
\end{tabular}

decrease, as expected as the severity of the T-bend reduces. Assuming a linear strain distribution through the blank thickness, the differences in the magnitudes of the major and minor strains strongly suggest that the neutral axis does not remain at mid-thickness as assumed by Ueda et al. and Wang et al. If the neutral axis did remain at mid-thickness, it would be expected that the maximum major strain would equal the maximum absolute minor strain.

The strain distributions along the mid-plane of the substrate (i.e. the strain versus curvilinear position) for T-bends from 0T to $4 \mathrm{~T}$ are shown in Fig. 11a and b for major and directional strains respectively. At low T-bend levels, the major strain is a maximum at the centre of the T-bend curve ( $225 \%$ at $0 \mathrm{~T}$, see Table 1). As the T-bend level and spacer thickness is increased, the major strain decreases, to $\sim 26 \%$ at $4 \mathrm{~T}$, and acts more uniformly over the bend length; the strains would be expected to continue decreasing with further increases in the T-bend level. A similar trend is observed in the directional strain results, albeit with smaller magnitudes initially and the maxima always acting in the centre. The maxima in the major and directional strain predictions converge at higher T-bend levels, which is explained by the strain distribution with changing curvature of the T-bend (as discussed below).

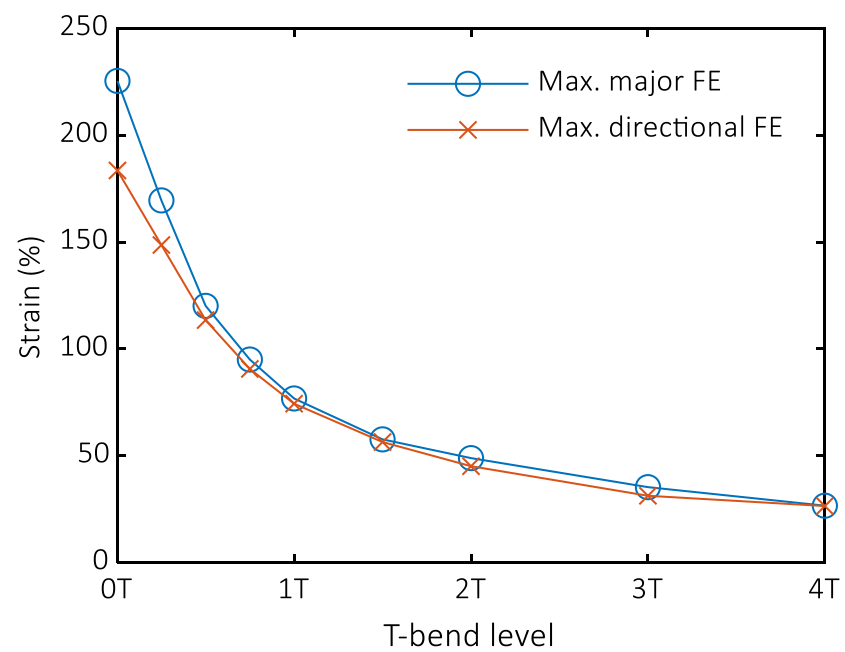

Fig. 10 Comparison of FE-predicted major and minor strains versus Tbend level 
Fig. 11 Predicted surface strains determined as a major strains and b directional strains versus the curvilinear position of the T-bend blank

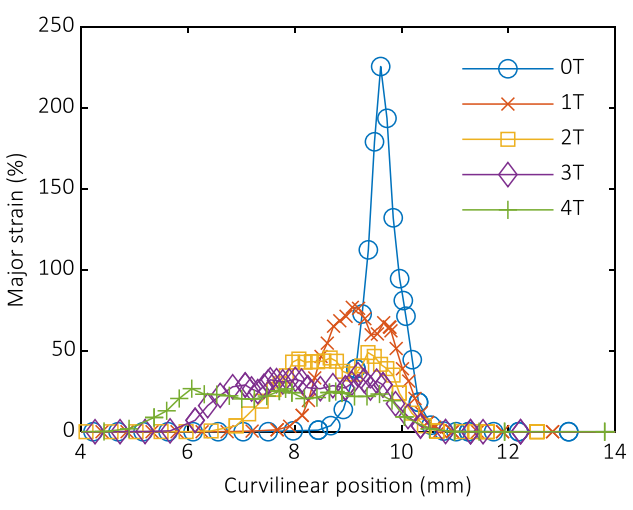

(a)

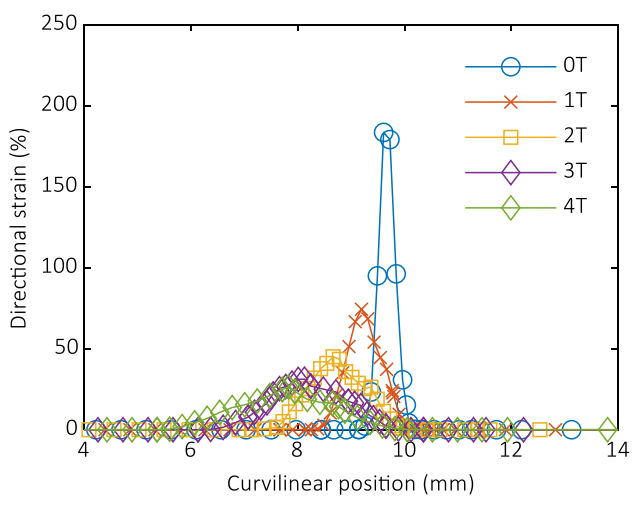

(b)
At $0 \mathrm{~T}$, the length of coating which is subjected to such large strains is relatively small $(\sim 0.5 \mathrm{~mm}$ FWHM), and it is seen that the length subjected to strain increases as the T-bend level increases ( $4.4 \mathrm{~mm} \mathrm{FWHM}$ at $4 \mathrm{~T})$ (see Table 2$)$. This results in coatings being subjected to strains of smaller magnitude but over larger lengths (see Fig. 11).

As the level of T-bend is increased, the radius of curvature increases and the sharpness of the curves decreases, resulting in lower strains. As the radius of curvature of the system increases (decrease in curvature), it tends closer towards a flat plane resulting in the system being strained in-plane. This explains why there is the convergence of the major and directional strains at higher T-bend levels. The FWHM crosssectional lengths over which the major strains act are larger than those over which the directional strains act (see Table 2). The major strain values account for the T-bend curvature and the resultant directionality of the strains, whilst the directional strain only determines the strain along one axis which can artificially reduce the strain values (see §2.4.2). Hence, the directional strains only show a high level of strain at one position, whilst the major strains show high strain values across the length of the curved surface. Away from the apex of the curve, there is a greater variation in the distance between points along the axis perpendicular to the surface and therefore, the distance between two adjacent points can no longer be assumed to be in the same plane.

Table 2 Full width at half maximum (FWHM) values of curvilinear position for the major and directional strain distributions predicted by FE at various T-bend levels

\begin{tabular}{llllll}
\hline Strain & \multicolumn{5}{l}{ FWHM curvilinear length (mm) } \\
\cline { 2 - 6 } & $0 \mathrm{~T}$ & $1 \mathrm{~T}$ & $2 \mathrm{~T}$ & $3 \mathrm{~T}$ & $4 \mathrm{~T}$ \\
\hline Major & 0.5 & 1.6 & 2.4 & 3.5 & 4.4 \\
Directional & 0.4 & 0.8 & 1.3 & 1.8 & 2.1 \\
\hline
\end{tabular}

The strain values across the width of the specimen are shown in Fig. 12. The variation of the major strain over the cross-sectional width for different T-bend levels is shown in Fig. 12a. As shown previously, the strains decrease with increasing T-bend level. Across the middle of the sample ( $2 \mathrm{~mm}$ to $\sim 8 \mathrm{~mm}$ ), there is little variation in the strain values, which shows that the strain values quoted at the mid-plane are representative of the sample. The edge effects are a result of the free surfaces at the material edges. The variation in the minor strain measurements are shown in Fig. 12b. Similar to the behaviour observed in the major strain, albeit compressive rather than tensile, the absolute minor strains (i.e. the maximum compressive strain) decrease with increasing T-bend levels. There is little variation across the middle of the samples.

The strain values decrease exponentially with increasing Tbend levels. MATLAB was used to fit a curve to the FE major strain $(\varepsilon)$ data (see Fig. 13) using:

$\varepsilon=a \cdot e^{-b \mathrm{x}}+c \cdot e^{-d \mathrm{x}}$

where $e$ is the natural exponent and $a, b, c$ and $d$ are fitted constants (see Table 3 ). A comparison is made between the FE-predicted and fitted data (see Fig. 13). There is good agreement between the two master curves. From here on the FE curve shown will be that of the fitted data.

\subsection{Analytical surface strains in T-bends}

The strains determined analytically using the Ueda et al. model (see Eq. (1)) and Wang et al. model (see Eq. (6)) are shown in Table 4. For both of the models, the analytically determined outer surface strains are high at 0T ( 92-100\%) and decrease to low levels $(\sim 20-22 \%)$ at $4 \mathrm{~T}$ (see Table 4$)$ ). At 0T, the Ueda et al. model predicts the largest strain (100\%), compared with 92\% from the Wang et al. model. The models converge and show good agreement at $\mathrm{T}$-bend levels greater than $\sim 0.75 \mathrm{~T}$ (see Table 4). 


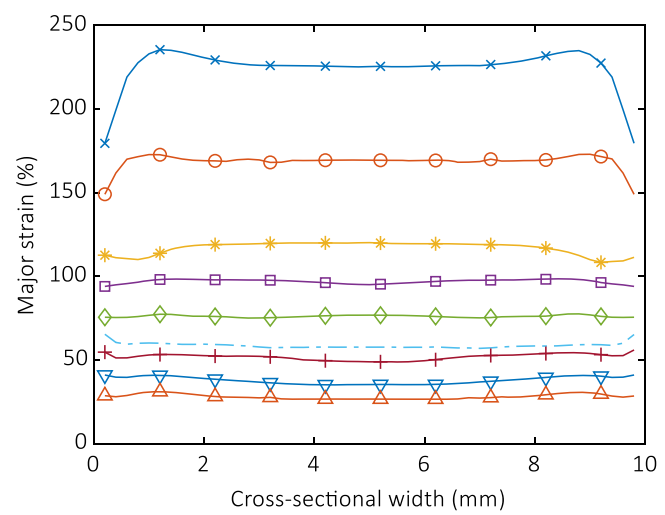

(a)

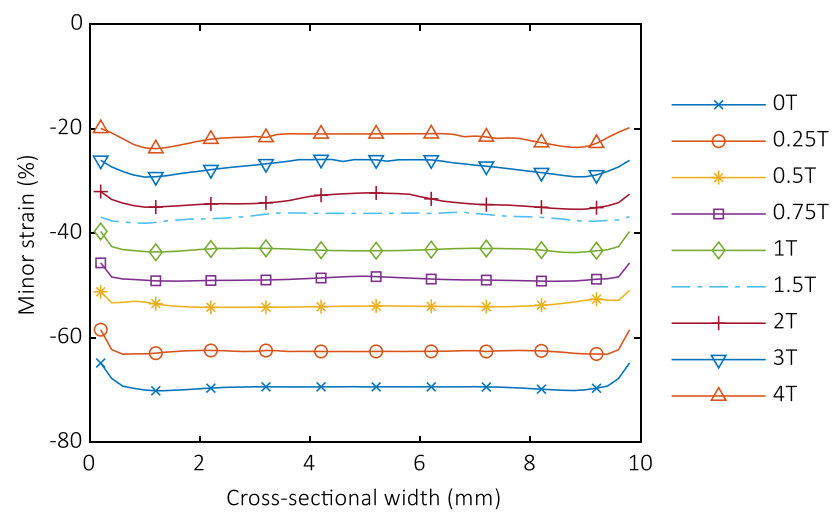

(b)

Fig. 12 Variation in a maximum major strain and $\mathbf{b}$ maximum compressive minor strain across the cross-sectional length

The analytically determined strains versus T-bend level are shown in Fig. 14. The trends observed are similar, showing an exponential decrease with an increasing level of T-bend. Although there are some small variations in the magnitudes of the calculated strains at low T-bend levels, the overall agreement between the data is good and as expected (decrease in strain with an increase in T-bend level).

The Ueda et al. and Wang et al. models are compared to the strain behaviour determined by FE modelling (see Fig. 14). The FE-predicted strains are much higher at low levels of Tbend, and although the strains do start to converge at higher levels of T-bend, they are still greater by $\sim 33 \%$ than the results predicted by the Ueda et al. and Wang et al. models. This suggests that the analytical models underestimate the surface strains of the T-bend test and that some of the assumptions made are an oversimplification.

The distribution of the major strains over the curvilinear distance (see Fig. 11a) predicted by FE showed that at low T-bend levels, the maximum strain effectively acts on a point position, whilst at high T-bend values, the strain magnitude is

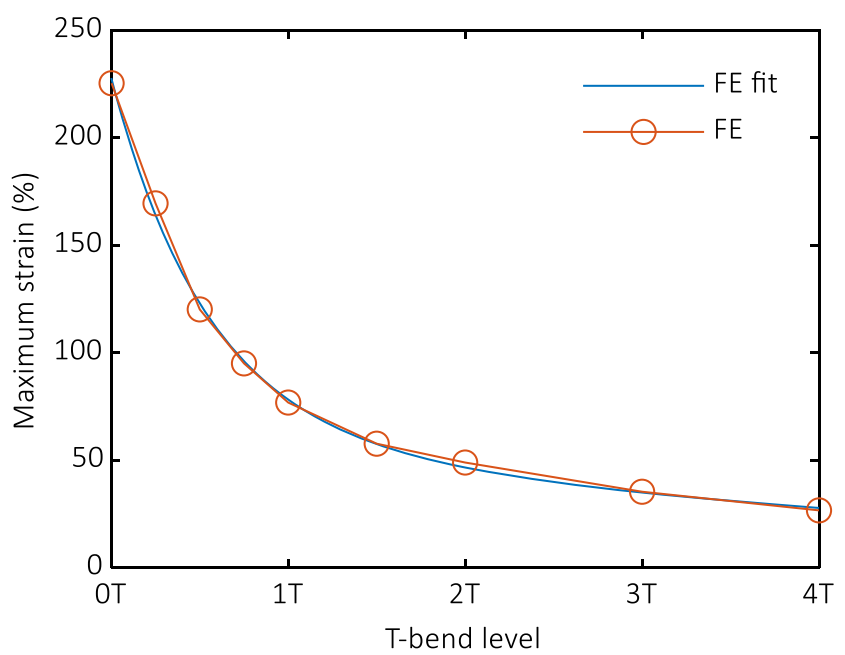

Fig. 13 Comparison of master curves showing the FE-predicted maximum major strain and exponentially fitted data versus T-bend level smaller and acts over a larger curvilinear length. The Ueda et al. and Wang et al. models do not account for this localisation and as such, the strain distribution appears to act equally over the face of the T-bend. Redistributing the FE strain to act over a rectangular area spanning $85 \%$ of the curved length reduces the FE strain magnitude and is representative of the assumption made by Ueda et al. (see Fig. 15a). The Ueda et al. strains agree well with these redistributed strains, and comparing the strain area of the FE data and the assumed area of the Ueda et al. strains support this hypothesis (see Fig. 15b) (the strain area refers to the area under the strain $(\%)$ versus curvilinear position (mm) curve (see Fig. 11) and as such, this area has units of $\mathrm{mm} \%$ ). This suggests that the FE predictions are correct. It should be noted that although Ueda et al. predicted maximum strains of $100 \%$ at $0 \mathrm{~T}$, only coatings with free film strain to failures greater than $\sim 200 \%$ were able to pass a $0 \mathrm{~T}$ T-bend test [12]. This suggests that the maximum applied strain in a $0 \mathrm{~T} \mathrm{~T}$-bend test is in fact much higher than the $100 \%$ predicted by Ueda et al. and is in the order of that predicted here by the FE model.

\subsection{Analytical model development}

The FE predictions show that the analytical models proposed by Ueda et al. and Wang et al. underestimate the maximum strains and do not fully capture the large strain behaviour at low T-bend levels. Both analytical models assume that the position of the neutral axis remains constant throughout the deformation process; however, the FE results show, given the large magnitudes of the surface strains, that the neutral axis

Table 3 Coefficients for FE data fitted to an exponential curve (see Eq. (11))

\begin{tabular}{lllll}
\hline Coefficient & $a$ & $b$ & $c$ & $d$ \\
\hline Value & 162.1 & 1.859 & 65.73 & 0.2167 \\
\hline
\end{tabular}


Table 4 Maximum analytically determined surface strains for $\mathrm{T}$ bends from $0 \mathrm{~T}$ to $4 \mathrm{~T}$ as proposed by the Ueda et al. [12] and Wang et al. [21]

\begin{tabular}{lllllllllll}
\hline Model & Profile & \multicolumn{1}{l}{ Maximum strain (\%) } & & & & & \\
\cline { 3 - 11 } & & $0 \mathrm{~T}$ & $0.25 \mathrm{~T}$ & $0.5 \mathrm{~T}$ & $0.75 \mathrm{~T}$ & $1 \mathrm{~T}$ & $1.5 \mathrm{~T}$ & $2 \mathrm{~T}$ & $3 \mathrm{~T}$ & $4 \mathrm{~T}$ \\
\hline Ueda et al. [12] & Semi-circular & 100 & 80 & 67 & 57 & 50 & 40 & 33 & 25 & 20 \\
Wang et al. [21] & Numerical (FE) & 92 & 64 & 57 & 54 & 46 & 42 & 36 & 27 & 22 \\
\hline
\end{tabular}

does move towards the inner surface of the blank. Movement of the neutral axis is a phenomenon which is well documented in the metal forming industry [22, 23], and empirical studies have been performed to predict k-factors [24] (position of the neutral axis expressed as a fraction of the thickness of the sheet, e.g. a k-factor of 0.5 refers to a neutral axis at the mid-plane of the material) for different degrees of bending. However, the use of established estimated k-factors is not sufficient to determine the surface strains in the T-bend test as they underestimate how much the neutral axis moves.

The radius of curvature of the neutral axis $\left(r_{n}\right)$ is typically smaller than the thickness of the sheet $(t)$ for $0 \mathrm{~T}$ to $2 \mathrm{~T}$, meaning that most theories or simplifications (such as simple bending theory) to describe bending are not valid as they require that $r_{\mathrm{n}}>5 \mathrm{t}$.

It is proposed to extend the theory in the Ueda et al. model by accounting for the movement of the neutral axis to predict the surface strains at the outer bend surface using Eq. (12) which is a combination of Eqs. (2) and (3), and the strains at the inner bend surface using Eq. (13) which is a combination of Eqs. (2) and (5):

$\varepsilon_{\text {Outer }}=\frac{\frac{N+2}{2} \cdot t-r_{\mathrm{n}}}{r_{\mathrm{n}}}=\frac{(N+2) \cdot \mathrm{t}}{2 \cdot r_{\mathrm{n}}}-1$

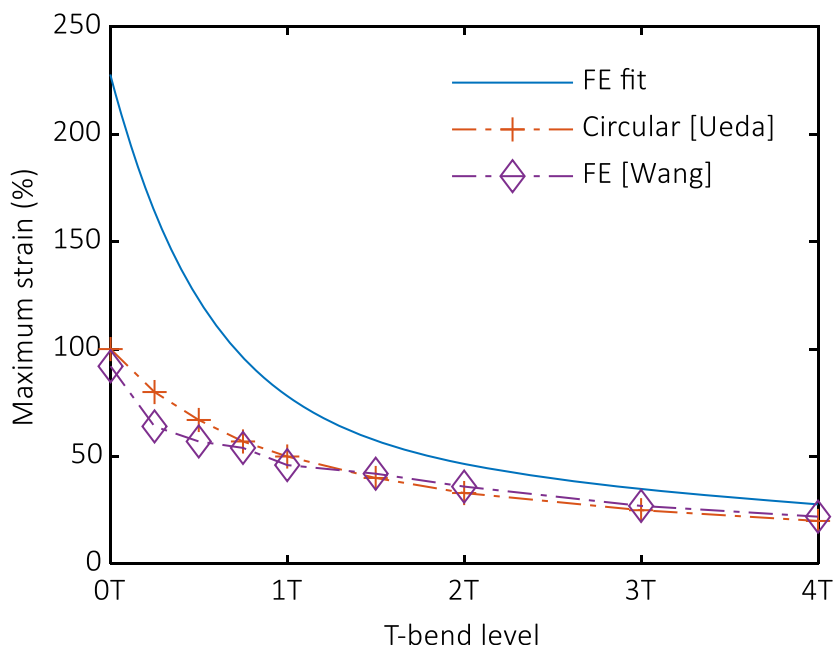

Fig. 14 Analytically determined strains versus T-bend level compared with the FE data $\varepsilon_{\text {Inner }}=\frac{\frac{N \cdot t}{2}-r_{\mathrm{n}}}{r_{\mathrm{n}}}=\frac{N \cdot t}{2 \cdot r_{\mathrm{n}}}-1$

where $r_{\mathrm{n}}$ is a simple function of the number of spacers $(N)$ and the substrate thickness $(t)$ :

$r_{\mathrm{n}}=f(N, t)$

\subsection{1 $r_{\mathrm{n}}$ determined by interpolation}

For simplicity, no thinning of the sheet is assumed. The FE model predicts a negligible amount of thinning, and consideration of the volume of material which is plastically deformed confirms that thinning effects are small. A linear strain distribution across the thickness of the substrate is also assumed to allow the position of the neutral axis (NA) and radius of curvature $r_{\mathrm{n}}$ to be predicted. Interpolation between the tensile strain at the outer surface and the compressive strain at the inner surface (i.e. over the substrate thickness, see Fig. 16) is used to determine the position through the sheet thickness at which the strain is $0 \%$.

The resultant strain values, from Eq. (12) using the interpolated values for $r_{\mathrm{n}}$, fit relatively well with the FE predictions (see Fig. 17, 'Interpolation' line), although this method overestimates the strains at small radii of curvature (0T, no spacer). It is clear from these results that the neutral axis does indeed move towards the inner radius. Hence, it is of interest to extend the Ueda et al. model to account for the movement of the neutral axis by deriving a simple function for $r_{\mathrm{n}}$ (see Eq. (14)).

\subsection{2 $r_{\mathrm{n}}$ determine by curved beam theory}

As suggested in Eq. (14), it is desirable to have a purely analytical relationship. In the analysis of curved beams (curved axis), it is generally accepted that the neutral axis is not located at the centroid of the member. Curved beam theory states that the 'resultant internal force caused by the stress distribution acting over the cross-section to be equal to zero' [25] from which a derivation for the position of the neutral axis from the centre of curvature $\left(r_{\mathrm{n}}\right)$ can be determined. Linear-elastic deformation is applied to the curved beam and as such, there is a linear stress distribution across the thickness: 


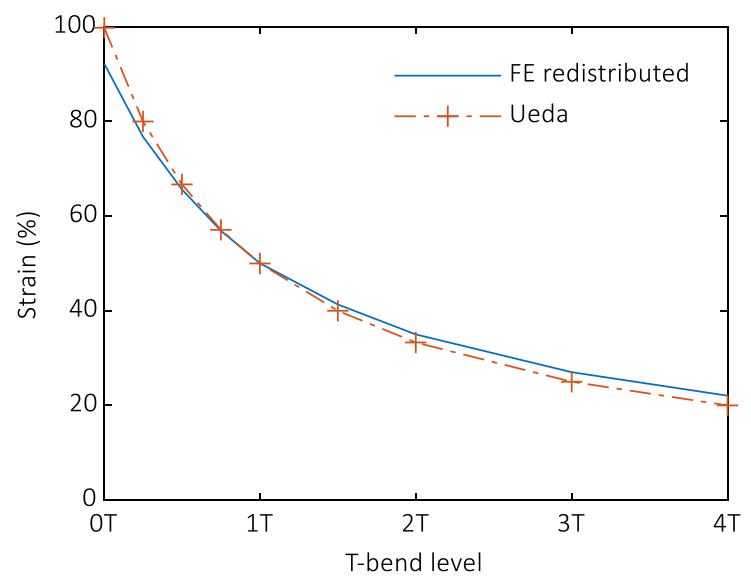

(a)

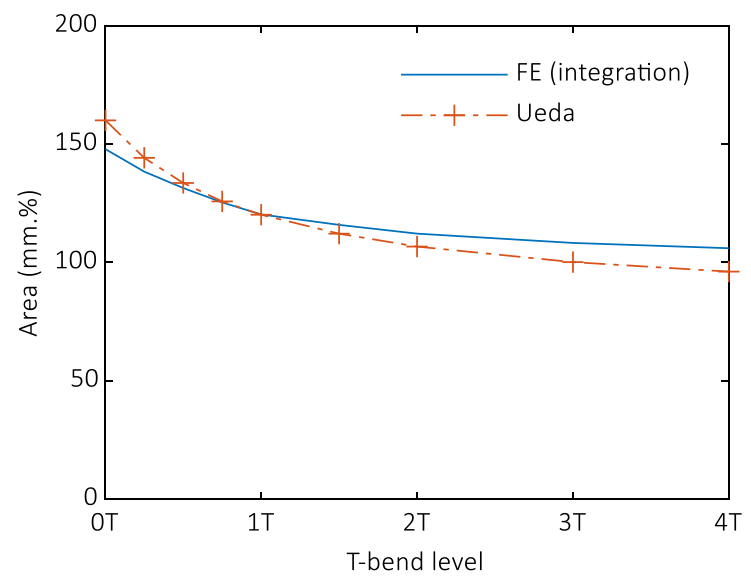

(b)

Fig. 15 a FE strains redistributed to act over $85 \%$ of the T-bend curvature length compared to the Ueda et al. predicted strains and $\mathbf{b}$ a comparison of the effective strain area which the FE and Ueda et al. predicted strains act over

$r_{\mathrm{n}}=\frac{A}{\int_{A} \frac{d A}{r}}$

$\int_{\mathrm{A}} \frac{\mathrm{dA}}{\mathrm{r}}=\mathrm{w} \cdot \ln \frac{\mathrm{r}_{\mathrm{o}}}{\mathrm{r}_{\mathrm{i}}}$

where $A$ is the area, $r$ is an arbitrary position of the element area, $r_{\mathrm{o}}$ is the outer radius and $r_{\mathrm{i}}$ is the inner radius.

Equation (15) can be simplified by combining Eqs. (3), (5) and (16) to:

$r_{\mathrm{n}}=\frac{t}{\ln \left(\frac{r_{\mathrm{o}}}{r_{\mathrm{i}}}\right)}=\frac{t}{\ln \left(\frac{N+2}{N}\right)}$

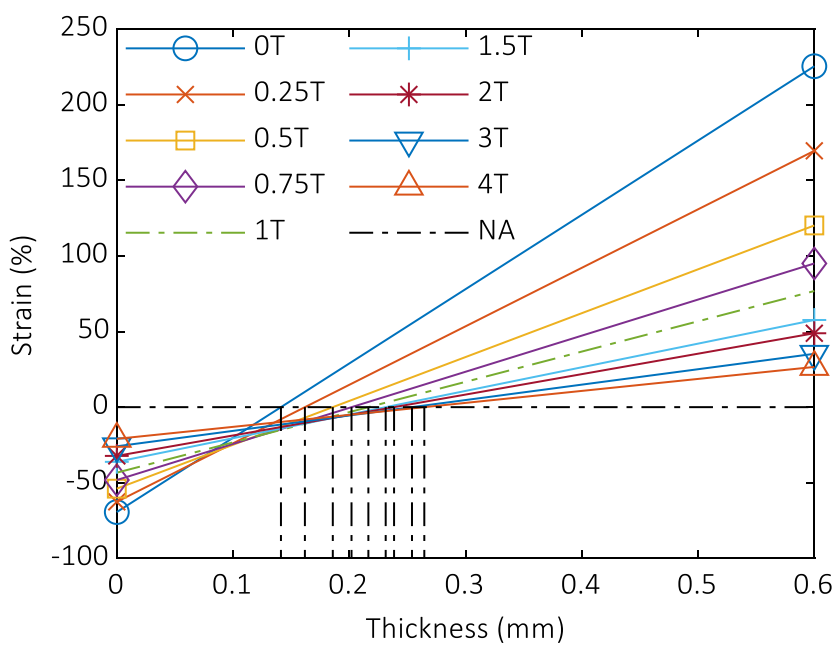

Fig. 16 Demonstration of how the position of the neutral axis (NA), indicated by the vertical black chain-dashed lines, is predicted by interpolation from the FE-predicted maximum tensile and compressive strains
Substituting Eq. (17) into Eq. (12) gives an expression for the outer surface strain of the T-bend which is solely dependent on the number of spacers (and hence the T-bend level):

$\varepsilon=\frac{N+2}{2} \cdot \ln \left(\frac{N+2}{N}\right)-1$

The strains predicted from curved beam theory using Eq. (18) are compared to the FE values in Fig. 18. The strain values show exponential decay with increasing T-bend level and are typically slightly smaller than the FE-predicted strains $(\sim 8 \%$ (absolute)).

As noted in $\S 3.2$, at low-level T-bends, e.g. 0T, the experimental profiles exhibit teardrops (see Fig. 9) meaning that the $r_{\mathrm{i}}$ is not $0 \mathrm{~mm}$ at $0 \mathrm{~T}$, but in this instance is in the order of

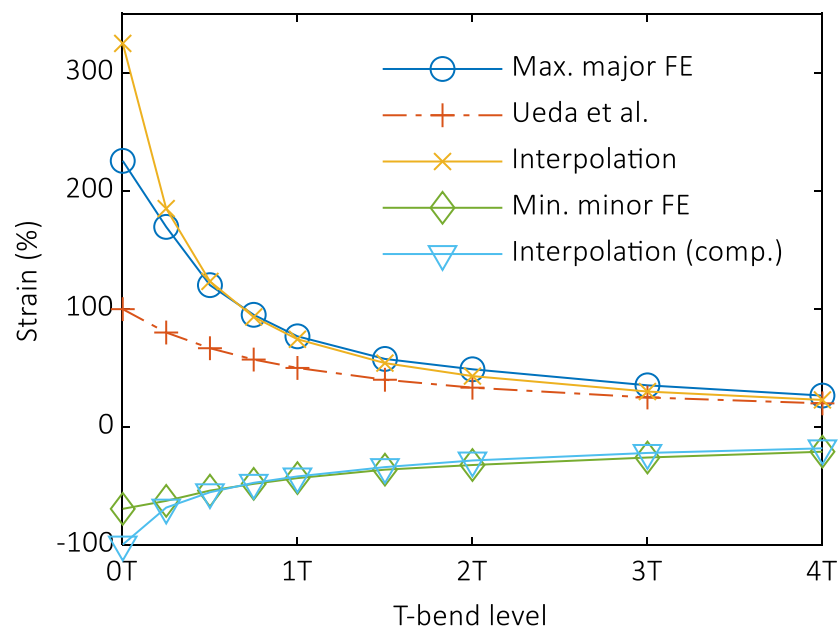

Fig. 17 Comparison of tensile surface strains predicted by FE (Max. major FE), by interpolating $r_{\mathrm{n}}$ (Interpolation) and the Ueda et al. model. The compressive strains predicted by FE (Min. minor FE) and by interpolating $r_{\mathrm{n}}$ (Interpolation (comp.)) versus T-bend level are also shown 


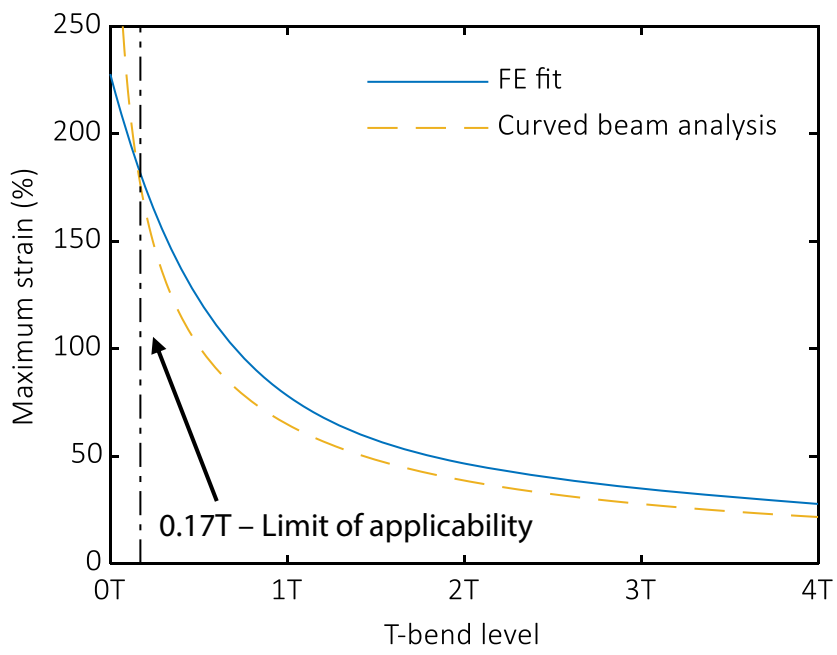

Fig. 18 Comparison of strains predicted using curved beam theory and FE data

$\sim 0.051 \mathrm{~mm}$ resulting in an actual T-bend of $0.17 \mathrm{~T}$. At very low T-bend levels $(<0.17 \mathrm{~T}$, the T-bend level at which the FE and calculated strains coincide, see Fig. 18), the calculated strains rapidly increase and tend towards infinity as the Tbend level approaches $0 \mathrm{~T}$. This result suggests that there is a limit of applicability to T-bend levels given material constraint limits which is in good agreement with the applicability requirements on the radius of curvature of the centroid $(R)$ and thickness $(t)$ specified by Young and Budynas [26] for analysis of curved beams:

$0.6<\frac{R}{t}<8$

The lower limit is equivalent to a minimum T-bend of $0.2 \mathrm{~T}$, which agrees well with the $0.17 \mathrm{~T}$ due to the teardrop formation. The upper limit is $15 \mathrm{~T}$, which is much larger than the Tbend levels typically applied.

\subsection{3 $r_{\mathrm{n}}$ determined by plastic bending of curved beams}

T-bend tests induce plastic deformation, whilst the abovecurved beam analysis assumes linear-elastic deformation. Johnson and Senior [27] investigated the plastic bending of heavily curved beams $\left(180^{\circ}\right.$ bend) with a trapezoidal crosssectional area (see Fig. 19).

The stress distribution in the beam is assumed to be fully plastic with equal plastic tensile and compressive stresses. An external load is applied to the beam ends and equilibrium conditions are applied to the curved beam. Moment equilibrium around the neutral axis is applied and reduces to:

$x^{3}-\frac{3}{2}[z \cdot(1-y)+1] \cdot x^{2}+\frac{3}{4} z \cdot\left(1+y^{2}\right)(1-y)+\frac{1}{4}\left[\left(1+3 y^{2}\right)-2 y^{3}\right]=0$

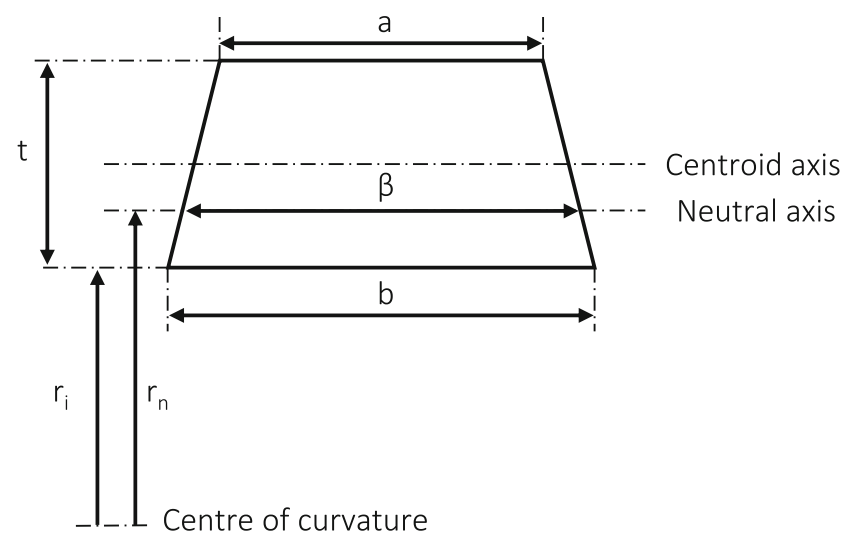

Fig. 19 Schematic showing trapezium cross-section

where $x, y$ and $z$ are defined as:

$x=\frac{\beta}{b} \quad y=\frac{a}{b} \quad z=\frac{r_{\mathrm{i}}}{t}$

where $\beta$ is the trapezium width at the location of the neutral axis, and $a$ and $b$ are the minor and major widths of the trapezium respectively (see Fig. 19). Solving for $x$ allows the radius of curvature of the neutral axis $\left(r_{\mathrm{n}}\right)$ to be determined using:

$r_{\mathrm{n}}=\frac{(b \cdot x-a)}{b-a} \cdot t+r_{\mathrm{i}}$

Combining Eq. (22) with Eq. (12) allows the outer surface strains of a T-bend test to be predicted. Equation (22) and the trapezoidal analysis cannot be solved when $a=b$ (perfectly rectangular); however, the analysis holds true as $a$ tends towards $b$. The surface strains of the T-bend tests were evaluated for a rectangular section (see Table 5) using:

$a=0.9999 \cdot b$

The predicted strains decrease with increasing T-bend level in an exponential manner. This is in good agreement with the FE predictions as shown in Fig. 20. The calculated strains follow a very similar trend to the FE strains.

Johnson and Senior [27] proposed an alternate form of Eq. (20) to account for when $a=b$ :

$z=\frac{\left(2 w^{2}-1\right)}{2 \cdot(1-2 w)}$

where $\mathrm{z}$ is defined as above (see Eq. (21)) and $w$ is:

Table 5 Surface strains calculated using Eqs. (12) and (22) to determine $r_{\mathrm{n}}$

\begin{tabular}{lccccc}
\hline T-bend & 0T & $1 \mathrm{~T}$ & $2 \mathrm{~T}$ & $3 \mathrm{~T}$ & $4 \mathrm{~T}$ \\
\hline Strain (\%) & 241 & 70 & 41 & 29 & 22 \\
\hline
\end{tabular}




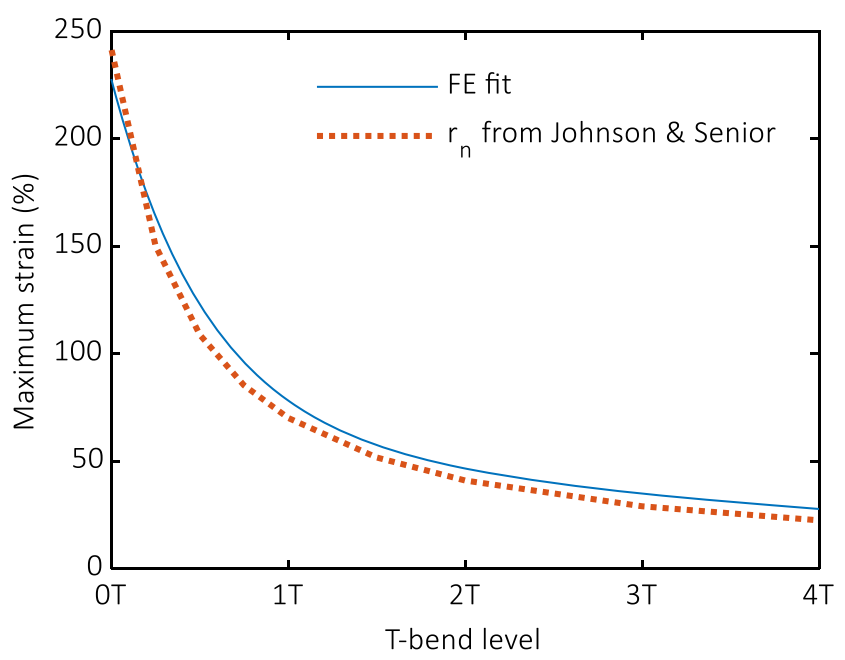

Fig. 20 Comparison between calculated surface strains calculated using Eqs. (12) and (22) to determine $r_{\mathrm{n}}$

$w=1-\frac{r_{\mathrm{n}}}{t}+\frac{N}{2}$

Solving for $w$ allows an analytical equation for $r_{\mathrm{n}}$ to be determined as a function of only sheet thickness and the number of spacers:

$r_{\mathrm{n}}=t \cdot\left((N+1)-\sqrt{\left(\frac{N+1}{2}\right)^{2}+0.25}\right)$

\subsubsection{New analytical model}

Combining Eqs. (12) and (26) gives a new simple analytical model for determining the surface strains in the T-bend test:

$\varepsilon=-\frac{1}{2} \cdot \frac{N-2 \sqrt{\left(\frac{N+1}{2}\right)^{2}+0.25}}{N+1-\sqrt{\left(\frac{N+1}{2}\right)^{2}+0.25}}$

The strains calculated using Eq. (27) are equal to the trapezoidal method. Hence, the outer surface strains can be easily determined for any given level of T. As expected, as $N$ tends to large values, and the curvature of the T-bend reduces, the position of the neutral axis tends towards the centroid axis. This new simple analytical equation (see Eq. (27)) allows the surface strains in the T-bend test to be calculated with minimal effort. There is excellent agreement with the FE data, as shown in Fig. 21.

\subsection{Experimentally determined surface strains}

The surface strains in the T-bend test were determined experimentally using the procedure outlined in $\$ 2.4$. The strain measurements determined at each T-bend level

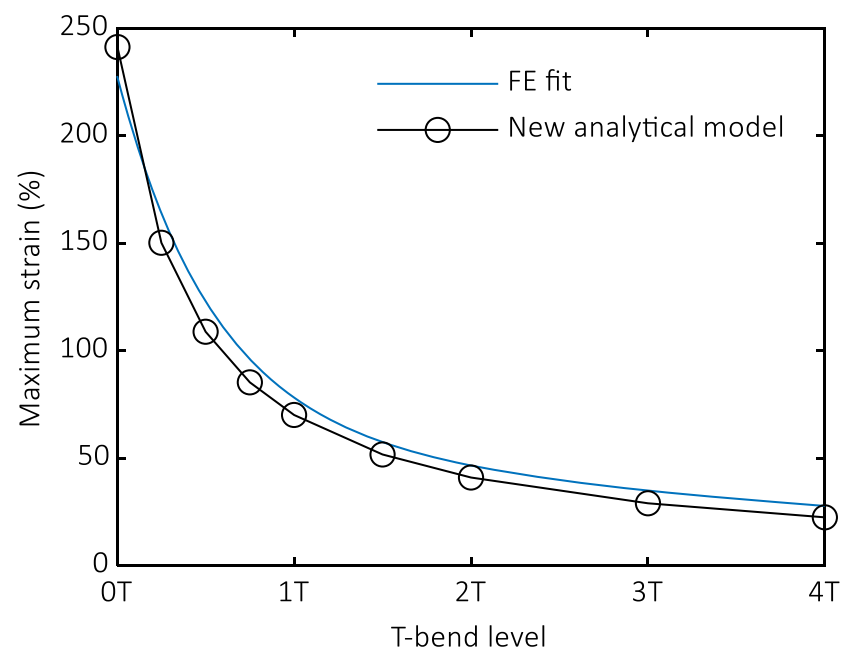

Fig. 21 Comparison of strains calculated using the new analytical model (Eq. (27)) and FE-predicted strains

were averaged to give single representative values of the strain for each T-bend level, ensuring that the lengths in which the data were averaged over were of a similar order to the FWHM strains distributions of the FE-predicted strains (see Fig. 11). The mean maximum strains determined for each level of T-bend are shown in Table 6. The experimental strain data show a decrease in strain with increasing T-bend level. The strains are initially high at $\sim 87 \%$ at $0 \mathrm{~T}$ (it is assumed that the strains are higher but significant cracking in the sample reduced the measured value) and decrease to $\sim 36 \%$ at $3 \mathrm{~T}$.

A comparison between the experimentally determined strains and the FE-predicted strains is shown in Fig. 22. An exponential decrease in the experimental strains from $1 \mathrm{~T}$ to $3 \mathrm{~T}$ is observed with magnitudes similar to the FE-predicted strains. The good agreement between the experimental and FE strains gives further confidence in the modelling work and strain predictions.

The mean experimental 0T strain does not follow the exponential trend. Given the high strain localisation in a $0 \mathrm{~T} \mathrm{~T}$ bend, the resolution of the measurement technique causes small errors in placement of the gridlines and the effective kerf of the gridline used to determine the strains to become significant. Due to the high strain at $0 \mathrm{~T}$, cracks in the coating and subsequent relaxation would also affect the strain measurement and effectively invalidate the 0T results. It was shown

Table 6 Mean maximum experimentally determined surface strains for $\mathrm{T}$-bends from $0 \mathrm{~T}$ to $3 \mathrm{~T}$

\begin{tabular}{lllll}
\hline T-bend & $0 \mathrm{~T}$ & $1 \mathrm{~T}$ & $2 \mathrm{~T}$ & $3 \mathrm{~T}$ \\
\hline Strain $(\%)$ & $>87^{*} \pm 13$ & $82 \pm 36$ & $46 \pm 21$ & $36 \pm 22$ \\
\hline
\end{tabular}

*Coating cracks significantly at $0 \mathrm{~T}$ 


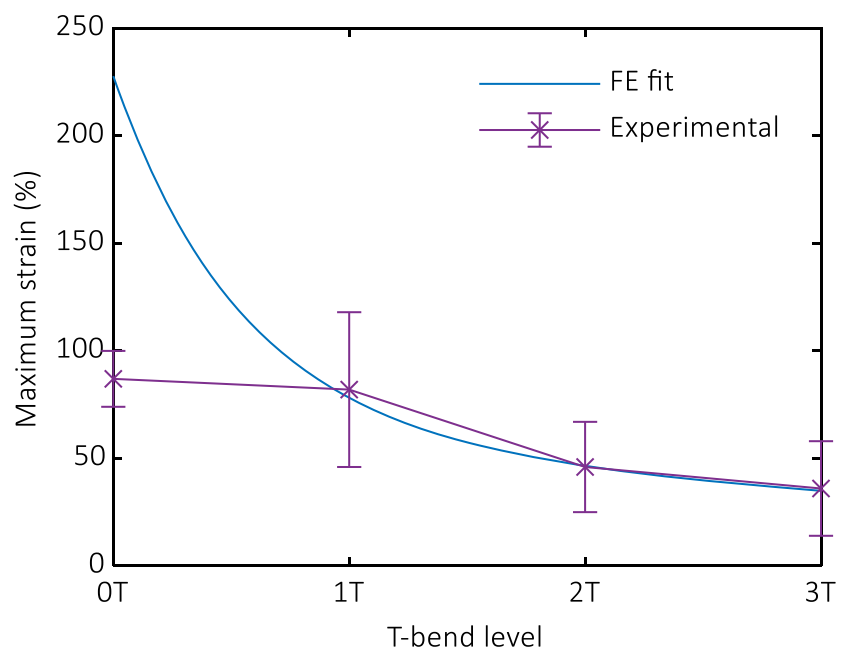

Fig. 22 Comparison of experimentally determined and FE-predicted strains versus T-bend level

in $\$ 3.3$ that the length over which the maximum strain acts increases as the level of T-bend increases. The maximum strain at $0 \mathrm{~T}$ was seen to act over an infinitesimally small area, significantly smaller than the quoted FWHM $(\sim 0.4 \mathrm{~mm}-$ $0.5 \mathrm{~mm}$, see Fig. 11); hence, it is believed that the technique used could not achieve a high enough resolution to determine the strains accurately at $0 \mathrm{~T}$.

At $1 \mathrm{~T}$, the size and number of the cracks were greatly reduced and have little effect on the strain measurement results. Higher level T-bends (2T and 3T) showed minimal cracking. The strain distribution in these higher T-bend levels is more uniform (see Fig. 11) across the T-bend which enables more accurate measurements.

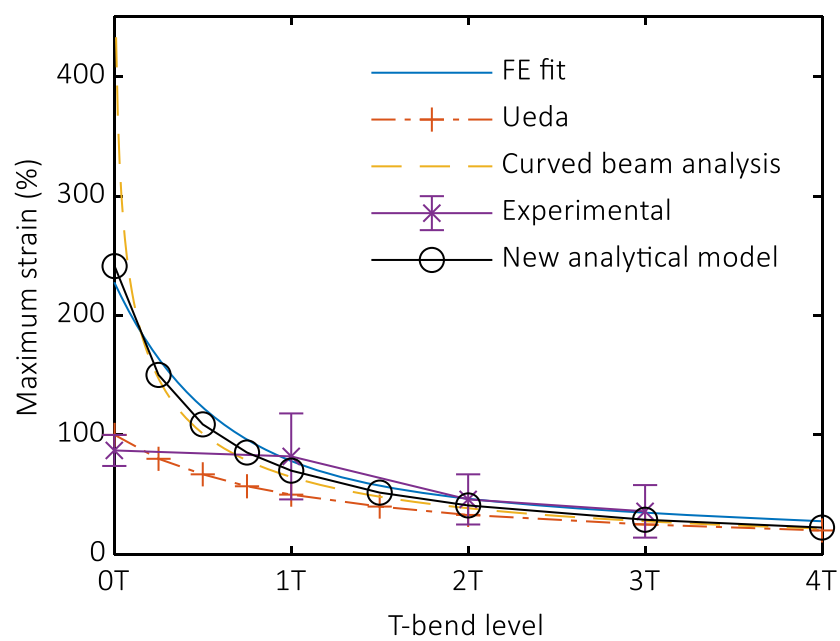

Fig. 23 Comparison of experimental, FE and analytically predicted maximum strains versus T-bend level

\subsection{Master curve comparison}

Master curves showing the maximum strains at the different levels of T-bend from the different analysis techniques are compared in Fig. 23 and Table 7. There is an exponential decrease in strain with an increase in T-bend level.

The Ueda et al. [12] model underestimates the strain at low T-bend levels due to the assumed uniform strain distribution (see §3.3), but shows better agreement with FE and experimental strains at higher T-bend levels. The experimental data show very good agreement with the FE predictions and analytical models at $\mathrm{T}$-bends $\geq 1 \mathrm{~T}$; the strain measurement is significantly lower at $0 \mathrm{~T}$ due to the strain acting in a highly localised manner (see §3.6). Despite this, the agreement with the FE strain values for T-bend levels greater than $1 \mathrm{~T}$ and validation provided by the excellent agreement between the profiles gives very good confidence to the results.

Preliminary FE modelling work investigating the effects of the substrate thickness, coefficient of friction between the blank and spacer, and the material properties has shown the surface strains in the T-bend test to be dependent only on the spacer thickness. These findings agree with the analytical models which do not include any material properties when determining the surface strains. This suggests that the Tbend test is independent of friction, substrate material thickness and properties, allowing the formability and critical forming strain of coatings to be easily extracted and compared irrespective of the metal substrate used. These findings are supported by the work done by Sorce [9]; however, it should be noted that experimental validation is required.

An accurate and simple new analytical model (see Eq. (27) and Table 7) to determine the surface strains in the T-bend test has been derived from the work of Johnson and Senior [27] and Ueda et al. [12]. This new analytical model shows excellent agreement with the FE predictions across the range of applied T-bends (see Table 7). This new analytical model quickly quantifies the T-bend test and improves the efficacy of the test by providing more useful and insightful data on the failure of the coatings and their formability.

\section{Conclusions}

The T-bend test is an industry standard comparative formability test. This work has quantified the surface strains experienced in T-bend tests from levels of 0T to 4T. A finite element model showed that for a 0T T-bend level the major strain reaches $225 \%$. The strains decrease exponentially with increasing T-bend level, declining to $27 \%$ at $4 \mathrm{~T}$. Analytical models to determine the surface strain were also examined. These models failed to adequately capture the localisation of the surface strain and gave large underestimates of the surface strain. Hence, a new simple analytical model was proposed for 
Table 7 Comparison of FE, experimental and analytical predicted maximum strains at varying $\mathrm{T}$-bend levels

\begin{tabular}{llllllllll}
\hline Strain type & \multicolumn{1}{l}{ Maximum strain (\%) } \\
\cline { 2 - 9 } & $0 \mathrm{~T}$ & $0.25 \mathrm{~T}$ & $0.5 \mathrm{~T}$ & $0.75 \mathrm{~T}$ & $1 \mathrm{~T}$ & $1.5 \mathrm{~T}$ & $2 \mathrm{~T}$ & $3 \mathrm{~T}$ & $4 \mathrm{~T}$ \\
\hline FE & 225 & 170 & 120 & 95 & 77 & 58 & 49 & 35 & 27 \\
Ueda et al. & 100 & 80 & 67 & 57 & 50 & 40 & 33 & 25 & 20 \\
Curved beam (elastic) & $839^{*}$ & 147 & 101 & 79 & 65 & 48 & 39 & 28 & 22 \\
Experimental & $>87^{+}$ & - & - & - & 82 & - & 46 & 36 & - \\
New analytical model & 241 & 150 & 109 & 85 & 70 & 52 & 41 & 29 & 22 \\
\hline
\end{tabular}

*Invalid

${ }^{+}$Significant cracking of the coating determining the surface strains in T-bend tests which shows excellent agreement with the FE predictions.

Increasing the level of T-bend increases the radius of curvature of the bend surface and hence reduces the surface strains experienced in the test. The experimentally measured and FE-predicted profiles of the T-bends showed excellent agreement giving confidence to the FE predictions. The experimentally determined surface strains showed very good agreement with the FE predictions for T-bend levels of $1 \mathrm{~T}$ and above.

It was also found that the surface strains in the T-bend test are independent of the substrate used. This means that the formability of coatings on different substrates can be easily compared and that the critical forming strain boundaries of a coating can be easily established. Hence, this work has revolutionised the T-bend test by quantifying the results, allowing the formability behaviour of coatings to be better understood. This ultimately will lead to enhanced and improved coatings to be developed in industry.

Availability of data and material The data used in this research is not publicly available due to ongoing studies.

Authors' contributions Fabian S. Sorce: conceptualisation, methodology, investigation, validation, software, formal analysis, data curation, writing - original draft, visualisation.

Sonny Ngo: conceptualisation, supervision.

Chris Lowe: conceptualisation, resources, writing-review and editing, supervision, funding acquisition.

Ambrose C. Taylor: conceptualisation, methodology, resources, writing - review and editing, supervision, funding acquisition.

Funding The authors would like to thank Becker Industrial Coatings Ltd. for funding this research.

\section{Compliance with ethical standards}

Conflict of interest The authors declare that they have no conflicts of interest.

Code availability The code used in this research is not publicly available due to ongoing studies.
Open Access This article is licensed under a Creative Commons Attribution 4.0 International License, which permits use, sharing, adaptation, distribution and reproduction in any medium or format, as long as you give appropriate credit to the original author(s) and the source, provide a link to the Creative Commons licence, and indicate if changes were made. The images or other third party material in this article are included in the article's Creative Commons licence, unless indicated otherwise in a credit line to the material. If material is not included in the article's Creative Commons licence and your intended use is not permitted by statutory regulation or exceeds the permitted use, you will need to obtain permission directly from the copyright holder. To view a copy of this licence, visit http://creativecommons.org/licenses/by/4.0/.

\section{References}

1. Grand View Reasearch Inc (2019) Pre-painted metal market size, share \& trends analysis report by product (Steel, Aluminum), by application (Construction, Transportation, Consumer Electronics), by region, and segment forecasts, 2019 - 2025, San Francisco

2. Sander, J. Coil coating. Hanover: Vincentz Network; 2014

3. British Standards Institution (2007) BS EN ISO 17132:2007. Paints and varnishes - T-bend test (ISO 17132:2007). BSI, London

4. Fletcher J, Walker J (2012) Flexibility and toughness. In: Koleske JV (ed) Paint and coating testing manual, Fifteenth edition of the Gardner-Sward handbook. West Conshocken: ASTM, pp 637-643

5. Yoshida K, Anyashiki T, Kakuchi T (2005) Synthesis and flexibility-hardness of polymer coatings based on melamine with mesogenic groups. Prog Org Coat 52(3):227-237. https://doi.org/ 10.1016/j.porgcoat.2005.01.001

6. Sorce FS, Shields T, Ngo S, Lowe C, Taylor AC (2020) The effect of varying molecular weight on the performance of HMMMcrosslinked polyester coatings. Prog Org Coat 149(105920). https://doi.org/10.1016/j.porgcoat.2020.105920

7. Kojima S, Watanabe Y (1996) Flexibility of epoxy coatings. Part 2: the relationship with the degree of cure. Polym Eng Sci 36(2):224 228. https://doi.org/10.1002/pen.10406

8. Giannakopoulos I (2012) The mechanical properties of polyesterbased coil coatings. Correlations with chemical structure $[\mathrm{PhD}]$. Imperial College London, London

9. Sorce FS (2020) Linking formability to the structure - property relationships of coil coatings $[\mathrm{PhD}]$. Imperial College London, London

10. Sorce FS, Ngo S, Lowe C, Taylor AC (2019) Quantification of coating surface strains in Erichsen cupping tests. J Mater Sci 54(10):7997-8009. https://doi.org/10.1007/s10853-019-03392-0 
11. Greunz T, Lowe C, Schmid M, Wallner GM, Strauß B, Stifter D (2019) Dry adhesion study of polyester/melamine clear coats on galvanized steel. Int J Adhes Adhes 95:102388. https://doi.org/10. 1016/j.ijadhadh.2019.05.005

12. Ueda K, Kanai H, Suzuki T, Amari T (2001) Effects of mechanical properties of paint film on the forming of pre-painted steel sheets. Prog Org Coat 43(4):233-242. https://doi.org/10.1016/S03009440(01)00199-0

13. Zhang K, Zhang K, Li HX, Chen GN (2009) Interface fracture behavior of electroplated coating on metal substrate under compressive strain. J Mater Process Technol 209(3):1337-1341. https://doi. org/10.1016/j.jmatprotec.2008.03.036

14. Kim S-R, Nairn JA (2000) Fracture mechanics analysis of coatingsubstrate systems - Part I - Analysis of tensile and bending experiments. Eng Fract Mech 65(5):573-593

15. Kim S-R, Nairn JA (2000) Fracture mechanics analysis of coatingsubstrate systems - Part II - Experiments in bending. Eng Fract Mech 65(5):595-607

16. Deflorian F, Fedrizzi L, Rossi S (2000) Effects of mechanical deformation on the protection properties of coil coating products. Corros Sci 42(7):1283-1301. https://doi.org/10.1016/S0010938X(99)00086-4

17. Tekkaya AE, Trompeter M, Pham HD (2010) On the reduction of the gloss property of organic coated sheet metal after forming. Prod Eng 4(5):483-489. https://doi.org/10.1007/s11740-010-0215-8

18. Behrens B-A, Hübner S, Gaebel CM (2017) Forming-induced gloss reduction of coil coated sheets for white goods. Procedia Eng 183: 107-112. https://doi.org/10.1016/j.proeng.2017.04.025
19. Lowe C (1995) Coil coating test methods and how some radiation curable coatings fare. Polym Paint Colour J 183:115-126

20. Cheong Z, Sorce FS, Ngo S, Lowe C, Taylor AC (2020) The effect of substrate material properties on the failure behaviour of coatings in the Erichsen cupping test. Prog Org Coat 151:106087. https://doi. org/10.1016/j.porgcoat.2020.106087

21. Wang Q, Dong X-H, Li H-Z, Zhang H-M (2011) Analytical model for straight hemming based on minimum energy method. J Zhejiang Univ Sci A 12(7):532-542. https://doi.org/10.1631/jzus. A1000458

22. Hosford WF, Caddel RM (2007) Metal forming: mechanics and metallurgy, Third edn. Cambridge University Press, Cambridge

23. Marciniak Z, Duncan JL, Hu SJ (2002) Mechanics of sheet metal forming, Second edn. Butterworth-Heinemann, Oxford

24. Benson S (2018) Analyzing the k-factor in sheet metal bending. Available from: https:/www.thefabricator.com/thefabricator/ article/bending/analyzing-the-k-factor-in-sheet-metal-bending. Accessed 08/05/2020

25. Hibbeler, RC (2011) Mechanics of materials, Eighth edn in SI Units. Prentice Hall, Singapore

26. Young WC, Budynas RG (2002) Roark's formulas for stress and strain, Seventh edn. McGraw-Hill, New York

27. Johnson W, Senior BW (1957) The plastic bending of heavily curved beams. J Roy Aeronaut Soc 61(654):824-830. https://doi. org/10.1017/S0368393100134807

Publisher's note Springer Nature remains neutral with regard to jurisdictional claims in published maps and institutional affiliations. 